\title{
On squares, outside guessing of clubs and $I_{<f}[\lambda]$
}

\author{
by
}

Mirna Džamonja and Saharon S helah (Jerusalem)

\begin{abstract}
Suppose that $\lambda=\mu^{+}$and $\mu$ is singular. We consider two aspects of the square property on subsets of $\lambda$. First, we have results which show e.g. that for $\aleph_{0}<\kappa=$ $\operatorname{cf}(\kappa)<\mu$, the equality $\operatorname{cf}([\mu] \leq \kappa, \subseteq)=\mu$ is a sufficient condition for the set of elements of $\lambda$ whose cofinality is $\leq \kappa$ to be split into the union of $\mu$ sets with squares. Secondly, we introduce a certain weak version of the square property and prove that if $\mu$ is a strong limit, then this weak square property holds on $\lambda$ without any additional assumptions.

In the second section we start with two universes $V_{1} \subseteq V_{2}$ of set theory, and a regular cardinal $\kappa$ in $V_{1}$ such that the cofinality of $\kappa$ in $V_{2}$ is $\theta<\kappa$. Assume $\kappa^{+}$is preserved and $\kappa$ is inaccessible in $V_{1}$ with $2^{\kappa}=\kappa^{+}$. We show that then there is an unbounded subset $C$ of $\kappa$ in $V_{2}$ such that for every club $E$ of $\kappa$ in $V_{1}$, the difference $C \backslash E$ is bounded. We have further results of a similar flavor. Some of our results were independently obtained by Moti Gitik, using different methods.

In the third section we consider the connection between the ideal $I[\lambda]$ and the notions of square and weak square. We show that these notions are a part of a larger family of properties which can all be introduced through a single definition of $I_{<f}[\lambda]$ by changing the parameter $f$. We discuss further properties of $I_{<f}[\lambda]$ and some other similarly defined notions. We have further results on $I[\lambda]$ in the last section.
\end{abstract}

0. Introduction. The problems studied in this paper come naturally in the study of cardinal arithmetic. The notions involved, like the ideal $I[\lambda]$, decomposition into sets with squares and club guessing, have been extensively investigated and applied by the second author in [Sh g] and related papers, both before and after [Sh g].

1991 Mathematics Subject Classification: 03E05, 03E99, 04A20.

Authors partially supported by the Basic Research Foundation Grant number 0327398 administered by the Israel Academy of Sciences and Humanities. The first author thanks the Hebrew University and the Lady Davis Foundation for the Forchheimer Postdoctoral Fellowship. For easier future reference, note that this is publication [DjSh 562] in Shelah's bibliography. The results presented were obtained in the period April to August 1994. The appendix was added in December 1994. We wish to thank Moti Gitik and Ofer Shafir for their interest and helpful comments, as well as James Cummings for pointing out a difficulty. 
In [Sh 351, §4] and [Sh 365, 2.14] it is shown that if $\mu$ is a regular cardinal, then $\left\{\alpha<\mu^{+}: \operatorname{cf}(\alpha)<\mu\right\}$ can be written as the union of $\mu$ sets on which there are squares. In 1.1 of the present paper it is shown that for a singular cardinal $\mu$ and $\aleph_{0}<\operatorname{cf}(\kappa)=\kappa<\mu$, if $\operatorname{cf}([\mu] \leq \kappa, \subseteq)=\mu$, then $\left\{\alpha<\mu^{+}: \operatorname{cf}(\alpha) \leq \kappa\right\}$ is the union of $\mu$ sets with squares. The proof is an application of [Sh 580]. The present result improves [Sh 237e, 2] for a singular $\mu$, as [Sh 237e, 2] had the same conclusion and assumed $\mu^{\leq \kappa}=\mu$. It also implies that under the assumptions of 1.1, the set $\left\{\alpha<\mu^{+}: \operatorname{cf}(\alpha) \leq \kappa\right\}$ is an element of $I\left[\mu^{+}\right]$, a fact which also follows from [Sh 420, 2.8]. Here $I\left[\mu^{+}\right]$is the ideal introduced in [Sh 108] or [Sh 88a].

Also in the first section there is a theorem which shows that if $\mu$ is a singular strong limit, then there is a weak version of the square principle, which we call square pretender, such that "many" elements of $\mu^{+}$have a club on which there is a square pretender. Moreover, all square pretenders in question can be enumerated in type $\mu$.

Suppose $\kappa$ is an inaccessible such that $2^{\kappa}=\kappa^{+}$and we change its cofinality to $\theta<\kappa$, so that $\kappa^{+}$is preserved. Then there is an unbounded subset $C$ of $\kappa$ in the extension, such that for every club $E$ of $\kappa$ in the ground model, $C \backslash E$ is bounded. This is one of the results of $\S 2$. We have further results of this nature, and with different assumptions. We shall refer to this type of results as to "outside guessing of clubs". Results on guessing clubs are reasonably well known (see [Sh g], [Sh e]). When Moti Gitik told the second author about his result quoted in A below, the second author was reminded of his earlier result quoted in B below, which was done in the preprint [Sh e], for a given club guessing. Note the connection between A and B via generic ultrapowers. The results of the form A are wider, as they also apply to presaturated ideals. It was then natural to try to prove such results using club guessing, and this is exactly what is done here.

We quote the theorems we referred to as $\mathrm{A}$ and $\mathrm{B}$ above:

Theorem A (Gitik) [Gi1, 2.1]. Let $V_{1} \subseteq V_{2}$ be two models of ZFC. Let $\kappa$ be a regular cardinal of $V_{1}$ which changes its cofinality to $\theta$ in $V_{2}$. Suppose that in $V_{1}$ there is an almost increasing (mod nonstationary) sequence of clubs of $\kappa$ of length $\chi$, with $\kappa^{+} \leq \chi$, such that every club of $\kappa$ of $V_{1}$ is almost included in one of the clubs of the sequence. Assume that $V_{2}$ satisfies the following:

(1) $\operatorname{cf}(\chi) \geq\left(2^{\theta}\right)^{+}$or $\operatorname{cf}(\chi)=\theta$.

(2) $\kappa \geq\left(2^{\theta}\right)^{+}$.

Then in $V_{2}$ there exists a sequence $\left\langle\tau_{i}: i<\theta\right\rangle$ cofinal in $\kappa$, consisting of ordinals of cofinality $\geq \theta^{+}$so that every club of $\kappa$ of $V_{1}$ contains a final segment of $\left\langle\tau_{i}: i<\theta\right\rangle$. 
Theorem B (Shelah) [Sh e, III, 6.2.B (old version)] = [Sh e, IV, 3.5 (new version)]. Let $\lambda$ be regular $>2^{\kappa}$ and $\kappa$ regular uncountable. Suppose that $S \subseteq\{\delta<\lambda: \operatorname{cf}(\delta)=\kappa\}$ is stationary and $I$ is a normal ideal on $\lambda$ such that $S \notin I$. If I is $\lambda^{+}$-saturated, then we can find a sequence (called a club system $)\left\langle C_{\delta}: \delta \in S\right\rangle$ such that each $C_{\delta}$ is a club of $\delta$ of order type $\operatorname{cf}(\delta)$, and for every club $C$ of $\lambda$ the set $\left\{\delta \in S: C_{\delta} \backslash C\right.$ is unbounded in $\left.\delta\right\} \in I$.

The proof of this theorem in fact gives that for every $S$ stationary in $\lambda$,

$(*)_{\lambda, S} \quad$ There exists $S_{1} \subseteq S$ stationary such that we can find a club system $\left\langle C_{\delta}: \delta \in S_{1}\right\rangle$ such that

$(\forall C$ a club of $\lambda)\left(\left\{\delta \in S_{1}: \delta>\sup \left(C_{\delta} \backslash C\right)\right\}\right.$ is not stationary $)$.

In the third section we unify the notions of square, weak square, silly square and $I[\lambda]$ by a single definition of $I_{<f}[\lambda]$, where $f$ is a parameter. We consider various properties of $I_{<f}[\lambda]$.

The last section is an appendix added after the paper was submitted. We prove in it two further theorems on $I[\lambda]$.

Before going on to the first section, we shall review some notation and conventions commonly used in the paper.

Notation 0.0. (0) Suppose that $\gamma \geq \theta$ and $\theta$ is a regular cardinal. Then

$$
S_{<\theta}^{\gamma}=\left\{\delta<\gamma: \aleph_{0} \leq \operatorname{cf}(\delta)<\theta\right\} .
$$

More generally, we use $S_{\mathrm{r} \theta}^{\gamma}$ for $\mathrm{r} \in\{<, \leq,=, \neq,>, \geq\}$ to describe

$$
S_{\mathrm{r} \theta}^{\gamma}=\left\{\delta<\gamma: \aleph_{0} \leq \operatorname{cf}(\delta) \& \operatorname{cf}(\delta) \mathrm{r} \theta\right\} .
$$

We use $S_{1}^{\gamma}$ to denote the set of successor ordinals below $\gamma$.

(1) For us an "inaccessible" is simply a regular limit cardinal $>\aleph_{0}$. Similarly to (0), we define

$$
S_{\lambda}^{\text {in }}=\{\mu<\lambda: \mu \text { is inaccessible and }<\lambda\} .
$$

(2) SING denotes the class of singular ordinals, that is, all ordinals $\delta$ with $\operatorname{cf}(\delta)<\delta$. REG is the class of regular cardinals.

(3) For $\lambda$ a regular cardinal $>\aleph_{0}$, we denote by $\operatorname{Club}(\lambda)$ the club filter on $\lambda$. The ideal of nonstationary subsets of $\lambda$ is denoted by NS[ $\lambda]$.

Sometimes we also speak of the club subsets of a $\lambda$ which do not obey the above restriction, but we shall point this out in each particular case.

(4) If $C \subseteq \lambda$, then

$$
\operatorname{acc}(C)=\{\alpha \in C: \alpha=\sup (C \cap \alpha)\} \quad \text { and } \operatorname{nacc}(C)=C \backslash \operatorname{acc}(C) \text {. }
$$

(5) If $\mathfrak{A}$ is a model on $\lambda$ and $a \subseteq \lambda$, then $\operatorname{Sk}_{\mathfrak{A}}(a)$ stands for the Skolem hull of $a$ in $\mathfrak{A}$.

(6) In the notation $\left\langle H(\chi), \in, \prec^{*}\right\rangle$, the symbol $\prec^{*}$ stands for the well ordering of $H(\chi)$.

(7) $J_{\kappa}^{\text {bd }}$ is the ideal of bounded subsets of $\kappa$, where $\kappa$ is a cardinal. 
1. On the square property. Our first concern is an instance of decomposing $S_{<\kappa}^{\mu^{+}} \cup S_{1}^{\mu^{+}}$into $\mu$ sets with squares, to be made more precise in a moment. We recall the definition of a square sequence on a set of ordinals:

Definition 1.0. Suppose that $S$ is a set of ordinals and $\kappa$ is an ordinal. The sequence $\bar{C}=\left\langle C_{\delta}: \delta \in S\right\rangle$ is a square on $S$ type-bounded by $\kappa$ iff the following holds for $\delta \in S$ :

(a) $C_{\delta} \subseteq \delta$ is closed.

(b) If $\delta$ is a limit ordinal, $C_{\delta}$ is unbounded in $\delta$.

(c) $\beta \in C_{\delta} \Rightarrow \beta \in S$.

(d) $\beta \in \operatorname{acc}\left(C_{\delta}\right) \Rightarrow C_{\beta}=C_{\delta} \cap \beta$.

(e) $\delta \in S \Rightarrow \operatorname{otp}\left(C_{\delta}\right)<\kappa$.

Theorem 1.1. Suppose that $\mu$ is singular, $\lambda=\mu^{+}$, and $\aleph_{0}<\operatorname{cf}(\kappa)=$ $\kappa<\mu$ is such that $\operatorname{cf}\left([\mu]^{\leq \kappa}, \subseteq\right)=\mu$. Then the set $\{\alpha<\lambda: \operatorname{cf}(\alpha) \leq \kappa\}$ is the union of $\mu$ sets with squares which are all type-bounded by $\kappa^{+}$.

Proof. It suffices to decompose $\lambda \backslash \mu$ into $\mu$ sets with squares. We shall fix a model $\mathfrak{A}=\left\langle H(\chi), \in, \prec^{*}\right\rangle$ for some large enough $\chi$.

For a moment, let us also fix an $a \in[\mu]^{\leq \kappa}$. We define

$$
X_{a}:=\left\{\gamma \in[\mu, \lambda): \operatorname{cf}(\gamma) \leq \kappa \& \operatorname{Sk}_{\mathfrak{A}}(a \cup\{\mu, \gamma\}) \cap \mu=a\right\} .
$$

We also define

$$
Y_{a, \gamma}:=\operatorname{Sk}_{\mathfrak{A}}(a \cup\{\mu, \gamma\}) \cap \gamma .
$$

It can be seen that the sets $\left\langle Y_{a, \gamma} \backslash \mu: \gamma \in X_{a}\right\rangle$ are quite close to a square sequence on $X_{a}$, but there is no reason to believe that the sets $Y_{a, \gamma}$ are closed. Note that there was a similar obstacle in [Sh 351]. Similarly to [Sh 351], we overcome this by defining inductively the following sets $X_{a}^{\oplus}$ and $Z_{a, \gamma}$.

For simplicity in notation, let us introduce

Definition 1.1.a. (1) Recall that a set $A$ of ordinals is said to be $\omega$-closed if

$$
\delta \in \operatorname{cl}(A) \& \operatorname{cf}(\delta)=\aleph_{0} \Rightarrow \delta \in A .
$$

We use cl to denote the ordinal closure.

(2) For a $\gamma \in[\mu, \lambda)$ with $\operatorname{cf}(\gamma)>\aleph_{0}$, a club $C$ of $\gamma$ is a-good if

$$
\beta_{1}<\beta_{2} \in C \& \operatorname{cf}\left(\beta_{1}\right)=\operatorname{cf}\left(\beta_{2}\right)=\aleph_{0} \Rightarrow Y_{a, \beta_{2}} \cap \beta_{1}=Y_{a, \beta_{1}},
$$

and

$$
\beta \in C \& \operatorname{cf}(\beta)=\aleph_{0} \Rightarrow \beta \in X_{a} \text { and } Y_{a, \gamma} \text { is } \omega \text {-closed. }
$$

Remark. Of course, we could without loss of generality assume that our language has a constant symbol for $\mu$, and so keep $\mu$ out of the definition of $X_{a}$ and $Y_{a, \gamma}$. We may skip $\mu$ from similar definitions later. 
We define inductively

$$
\begin{aligned}
X_{a}^{\oplus}:=\{\gamma \in[\mu, \lambda): & \operatorname{cf}(\gamma)=\aleph_{0} \& \gamma \in X_{a} \& Y_{a, \gamma} \text { is } \omega \text {-closed } \\
& \vee \kappa \geq \operatorname{cf}(\gamma)>\aleph_{0} \& \text { there is an } a \text {-good club } C \subseteq \gamma \\
& \&\left(\operatorname{cf}(\delta)=\aleph_{0} \& \delta \in C\right) \Rightarrow \delta \in X_{a}^{\oplus} \\
& \left.\vee \operatorname{cf}(\gamma)=1 \& \text { there is a limit } \delta>\gamma \text { with } \delta \in X_{a}^{\oplus}\right\} .
\end{aligned}
$$

For $\gamma \in X_{a}^{\oplus}$ we define inductively

$$
Z_{a, \gamma}:= \begin{cases}\operatorname{cl}\left(Y_{a, \gamma} \backslash \mu\right) \cap \gamma & \text { if } \operatorname{cf}(\gamma)=\aleph_{0}, \\ \bigcap_{C a \text {-good club of } \gamma} \bigcup_{\beta \in C \& \operatorname{cf}(\beta)=\aleph_{0}} Z_{a, \beta} & \text { if } \kappa \geq \operatorname{cf}(\gamma)>\aleph_{0} \text { and } \gamma \in X_{a}^{\oplus}, \\ Z_{a, \delta} \cap \gamma & \text { if } \operatorname{cf}(\gamma)=1 \text { and } \\ & \delta \in X_{a}^{\oplus} \text { is the minimal such limit }>\gamma .\end{cases}
$$

We show that $\left\langle Z_{a, \gamma}: \gamma \in X_{a}^{\oplus}\right\rangle$ is a square sequence on $X_{a}^{\oplus}$. As $a$ is going to be fixed for some time, we may slip and say "good" rather than $a$-good in the following.

Although we for most of the argument scholastically keep the $\bigcap$ over the good clubs of $\gamma$ in the definition of $Z_{a, \gamma}$ for $\gamma$ of uncountable cofinality, we invite the reader to check that any two good clubs of $\gamma$ give the same value to $\bigcup$ of the relevant $Z_{a, \beta}$. Hence we are in no danger of intersecting too many sets. This argument in particular shows that $Z_{a, \gamma}$ for such $\gamma$ is closed and unbounded in $\gamma$. Also note that in our definition of squares, successor ordinals play no role, so the decision of what to put as $Z_{a, \gamma}$ for a successor $\gamma$ is quite arbitrary.

FACT 1.1.b. Suppose that $\gamma \in X_{a}^{\oplus}$ and $\beta \in Z_{a, \gamma}$. Then:

(1) $\beta \in X_{a}^{\oplus}$.

(2) If $\beta \in \operatorname{acc}\left(Z_{a, \gamma}\right)$, then $Z_{a, \beta}=Z_{a, \gamma} \cap \beta$.

(3) $\gamma$ a limit ordinal $\Rightarrow \sup \left(Z_{a, \gamma}\right)=\gamma$.

(4) $\operatorname{otp}\left(Z_{a, \gamma}\right)<\kappa^{+}$.

(5) $Z_{a, \gamma}$ is closed.

Proof. (1)-(2) We prove the first two items together by induction on $\gamma$, dividing the discussion into several cases.

Case I: $\operatorname{cf}(\beta)=\operatorname{cf}(\gamma)=\aleph_{0}$.

(1)-(2) Since $\beta \in Z_{a, \gamma}=\operatorname{cl}\left(Y_{a, \gamma} \backslash \mu\right) \cap \gamma$, and $\operatorname{cf}(\beta)=\aleph_{0}$, by the $\omega$-closure of $Y_{a, \gamma}$, we deduce that $\beta \in Y_{a, \gamma}$. So, $\operatorname{Sk}_{\mathfrak{A}}(a \cup\{\mu, \beta\}) \subseteq \operatorname{Sk}_{\mathfrak{A}}(a \cup\{\mu, \gamma\})$, hence $Y_{a, \beta} \subseteq Y_{a, \gamma} \cap \beta$, and also

$$
a \subseteq \operatorname{Sk}_{\mathfrak{A}}(a \cup\{\mu, \beta\}) \cap \mu \subseteq \operatorname{Sk}_{\mathfrak{A}}(a \cup\{\mu, \gamma\}) \cap \mu=a .
$$

So, $\beta \in X_{a}$. We now show $Y_{a, \beta}=Y_{a, \gamma} \cap \beta$, from which it also follows that $Y_{a, \beta}$ is $\omega$-closed, hence $\beta \in X_{a}^{\oplus}$. 
We already know that $Y_{a, \beta} \subseteq Y_{a, \gamma} \cap \beta$. Now we proceed as in [Sh 430, 1.1]. In $\mathfrak{A}$ we can define just from $\mu, \beta$ a 1-1 onto function $f: \mu \rightarrow \beta$, as $\beta \in\left[\mu, \mu^{+}\right)$. The $\prec^{*}$-first such function, say $f^{*}$, is in $\operatorname{Sk}_{\mathfrak{A}}(a \cup\{\mu, \beta\})$, and also in $\operatorname{Sk}_{\mathfrak{A}}(a \cup\{\mu, \gamma\})$, since this set contains $\beta$. In $\operatorname{Sk}_{\mathfrak{A}}(a \cup\{\mu, \gamma\})$, this function is 1-1 from $a$ onto $\operatorname{Sk}_{\mathfrak{A}}(a \cup\{\mu, \gamma\}) \cap \beta$. In $\operatorname{Sk}_{\mathfrak{A}}(a \cup\{\mu, \beta\})$, the range of this function is $Y_{a, \beta}$. Since $f^{*}$ is a fixed function, we conclude that $Y_{a, \beta}=Y_{a, \gamma} \cap \beta$.

So,

$$
Z_{a, \beta}=\operatorname{cl}\left(Y_{a, \beta} \backslash \mu\right) \cap \beta=\operatorname{cl}\left(Y_{a, \gamma} \cap \beta \backslash \mu\right) \cap \beta=\operatorname{cl}\left(Y_{a, \gamma} \backslash \mu\right) \cap \beta=Z_{a, \gamma} \cap \beta .
$$

Case II: $\operatorname{cf}(\beta)=\aleph_{0} \& \operatorname{cf}(\gamma) \in\left(\aleph_{0}, \kappa\right]$.

(1) Let $C \subseteq \gamma$ be an $a$-good club of $\gamma$. By the definition of $Z_{a, \gamma}$, there is a $\delta \in C$ with $\operatorname{cf}(\delta)=\aleph_{0}$ such that $\beta \in Z_{a, \delta}$. By the first case, $\beta \in X_{a}^{\oplus}$.

(2) For any $a$-good club $C$ of $\gamma$, let us denote by $\delta_{C}$ the minimal element $\delta$ of $C$ with $\operatorname{cf}(\delta)=\aleph_{0}$ such that $\beta \in Z_{a, \delta}$. Note that $\delta_{C}$ is well defined (by the definition of $\left.Z_{a, \gamma}\right)$, and $\delta_{C}>\beta$. Then

$$
\begin{aligned}
& Z_{a, \gamma} \cap \beta= \bigcap_{C a-\text { good club } \subseteq \gamma}\left[\bigcup_{\delta \in C \cap \delta_{C} \& \operatorname{cf}(\delta)=\aleph_{0}}\left(Z_{a, \delta} \cap \beta\right)\right. \\
&\left.\cup \bigcup_{\delta \in C \backslash \delta_{C} \& \operatorname{cf}(\delta)=\aleph_{0}}\left(Z_{a, \delta} \cap \beta\right)\right] \\
&=\bigcap_{C a-\text { good club } \subseteq \gamma}\left[\bigcup_{\delta \in C \cap \delta_{C} \& \operatorname{cf}(\delta)=\aleph_{0}}\left(Z_{a, \delta_{C}} \cap \delta \cap \beta\right)\right. \\
&\left.\cup \bigcup_{\delta \in C \backslash \delta_{C} \& \operatorname{cf}(\delta)=\aleph_{0}}\left(Z_{a, \delta} \cap \delta_{C} \cap \beta\right)\right] \\
&=\bigcup_{C a-\text { good club } \subseteq \gamma}\left[Z_{a, \beta} \cap \delta \bigcup Z_{a, \beta}\right]=Z_{a, \beta} .
\end{aligned}
$$

Cas e III: $\operatorname{cf}(\beta) \in\left(\aleph_{0}, \kappa\right] \& \operatorname{cf}(\gamma)=\aleph_{0}$.

(1) Then $\beta \in \operatorname{cl}\left(Y_{a, \gamma} \backslash \mu\right) \cap \gamma$. Let $A$ be an unbounded subset of $\beta$ with $A \subseteq Y_{a, \gamma} \backslash \mu$, and let $C$ be the ordinal closure of $A$ in $\beta$. Hence $C$ is a club of $\beta$ and

$$
\delta \in C \& \operatorname{cf}(\delta)=\aleph_{0} \Rightarrow \delta \in Y_{a, \gamma} \backslash \mu .
$$

Suppose $\beta_{1}<\beta_{2} \in C$ and $\operatorname{cf}\left(\beta_{1}\right)=\operatorname{cf}\left(\beta_{2}\right)=\aleph_{0}$. By the first case, we know that $\beta_{1}, \beta_{2} \in X_{a}^{\oplus}$ and

$$
Y_{a, \beta_{1}}=Y_{a, \gamma} \cap \beta_{1}=Y_{a, \gamma} \cap \beta_{2} \cap \beta_{1}=Y_{a, \beta_{2}} \cap \beta_{1} .
$$

Hence $C$ is a good club of $\beta$ and $\beta \in X_{a}^{\oplus}$.

(2) Suppose that $E$ is a good club of $\beta$ and $\delta \in E$ has cofinality $\aleph_{0}$. Without loss of generality, $E \subseteq C$, where $C$ is as in the proof of (1) above. Hence 
$\delta \in Z_{a, \gamma}$, so by the first case, $Z_{a, \delta}=Z_{a, \gamma} \cap \delta$. Thus $\bigcup_{\delta \in E \& \operatorname{cf}(\delta)=\aleph_{0}} Z_{a, \delta}=$ $Z_{a, \gamma} \cap \beta$. Hence $Z_{a, \beta}=Z_{a, \gamma} \cap \beta$.

Case IV: $\operatorname{cf}(\beta), \operatorname{cf}(\gamma) \in\left(\aleph_{0}, \kappa\right]$.

(1) Let $C$ be a good club of $\gamma$; then there is a $\delta \in C$ with $\operatorname{cf}(\delta)=\aleph_{0}$ and $\beta \in Z_{a, \delta}$. By Case III, $\beta \in X_{a}^{\oplus}$.

(2) Suppose $E$ is a good club of $\gamma$. Let $\delta_{E} \in E$ be such that $\beta \in Z_{a, \delta_{E}}$ and $\operatorname{cf}\left(\delta_{E}\right)=\aleph_{0}$. Then, as in Case III, we can find an $a$-good club $C$ of $\beta$ such that $\left(\varepsilon \in C \& \operatorname{cf}(\varepsilon)=\aleph_{0}\right) \Rightarrow \varepsilon \in Y_{a, \gamma}$. In particular (by the first case), for any $\varepsilon \in C$ with $\operatorname{cf}(\varepsilon)=\aleph_{0}$, we have $Z_{a, \varepsilon}=Z_{a, \delta_{E}} \cap \varepsilon$. Hence $\bigcup\left\{Z_{a, \varepsilon}: \varepsilon \in C \& \operatorname{cf}(\varepsilon)=\aleph_{0}\right\}=Z_{a, \delta_{E}} \cap \beta$. By the third case, this is equal to $Z_{a, \gamma} \cap \beta$. In our calculation of $Z_{a, \beta}$ we can without loss of generality restrict ourselves to the good clubs of $\beta$ which are subsets of $C$. Hence $Z_{a, \beta}=Z_{a, \gamma} \cap \beta$.

Case V: $\operatorname{cf}(\beta)=1 \& \operatorname{cf}(\gamma) \in\left[\aleph_{0}, \kappa\right]$.

(1) By the definition of $X_{a}^{\oplus}$, we have $\beta \in X_{a}^{\oplus}$.

(2) Does not apply.

Case VI: $\operatorname{cf}(\beta)=\operatorname{cf}(\gamma)=1$.

(1) Let $\delta$ be a limit ordinal $>\gamma$ such that $\delta \in X_{a}^{\oplus}$. Then $\beta<\delta$, so $\beta \in X_{a}^{\oplus}$.

(2) Does not apply.

Cas e VII: $\operatorname{cf}(\beta)>1 \& \operatorname{cf}(\gamma)=1$.

(1) Let $\delta>\gamma$ be a limit ordinal such that $Z_{a, \gamma}=Z_{a, \delta} \cap \gamma$, and use Case V.

(2) If $\beta \in \operatorname{acc}\left(Z_{\gamma}\right)$, then $\beta \in \operatorname{acc}\left(Z_{\delta}\right)$, so $Z_{\beta}=Z_{\delta} \cap \beta=Z_{\gamma} \cap \beta$, by previous cases.

We proceed to the proof of (3)-(5).

(3) Suppose that $\gamma$ is a limit ordinal. First suppose that $\operatorname{cf}(\gamma)=\aleph_{0}$. In $\mathfrak{A}$, there is a cofinal function $f: \aleph_{0} \rightarrow \gamma$, definable from $\gamma$ only. Hence the first such $f$ is an element of $Y_{a, \gamma}$. Then $\operatorname{Ran}(f)$ is an unbounded subset of $\gamma \cap Y_{a, \gamma}, \operatorname{sosup}\left(Z_{a, \gamma}\right) \geq \sup \left(Y_{a, \gamma} \cap \gamma\right)=\gamma$.

If $\operatorname{cf}(\gamma)>\aleph_{0}$, then we know that for every $\delta \in Z_{a, \gamma}$ with $\operatorname{cf}(\delta)=\aleph_{0}$ we have $\delta=\sup \left(Z_{a, \delta}\right)$, so the conclusion follows from the definition of $Z_{a, \gamma}$.

(4) This follows since $\left|Y_{a, \beta}\right| \leq \kappa$ for any $\beta \in X_{a}$, so putting all the definitions together, $\left|Z_{a, \gamma}\right| \leq \kappa$.

(5) If $\operatorname{cf}(\gamma)=\aleph_{0}$, this is implicitly stated in the definition. Next, it is easy to check that for $\operatorname{cf}(\gamma)>\aleph_{0}$ (see the paragraph before Fact 1.1.b). Finally, for $\gamma$ a successor, we also obtain a closed set via our definition of $Z_{a, \gamma} \cdot \mathbf{- 1 . 1 . b}$

So we have now to prove that we can choose $\mu$ many $a$ such that all $\gamma \in[\mu, \lambda)$ of cofinality $\leq \kappa$ are in some $X_{a}^{\oplus}$. We shall use the following 
theorem of Saharon Shelah, which is a consequence of Theorem 1.4 of [Sh 580 ]. We note that in fact a stronger version of Theorem $\mathrm{C}$ follows from [Sh $580,1.4]$, where $\mu$ is not required to be a singular $>\kappa$, but just to be above some finitely many cardinal successors of $\kappa$.

Theorem C (Shelah). Suppose that $\kappa$ and $\mu$ are as above. Then there is $a \mathcal{P} \subseteq[\mu] \leq \kappa$ such that $|\mathcal{P}|=\mu$ and for every large enough $\theta$ and $x \in H(\theta)$, we can find a continuously increasing sequence $\bar{N}=\left\langle N_{i}: i\left\langle\kappa^{+}\right\rangle\right.$such that:

- $N_{i} \in N_{i+1}$ for $i \leq \kappa^{+}$.

- $x \in N_{i} \prec\left\langle H(\theta), \in, \prec^{*}\right\rangle$ for all $i$.

- $\left|N_{i}\right|=\kappa$ for all $i$.

- For every club $E$ of $\kappa^{+}$, there is an $i \in E$ such that $N_{i} \cap \mu \in \mathcal{P}$.

Now we fix a $\mathcal{P} \subseteq[\mu]^{\leq \kappa}$ as in Theorem $\mathrm{C}$, so, in particular, $|\mathcal{P}|=\mu$. We claim that $\bigcup\left\{X_{a}^{\oplus}: a \in \mathcal{P}\right\}$ contains all $\gamma \in[\mu, \lambda)$ with $\operatorname{cf}(\gamma) \leq \kappa$. This suffices, as we have just proved that $\left\langle Z_{a, \gamma}: \gamma \in X_{a}^{\oplus}\right\rangle$ is a square sequence, for any $a \in[\mu]^{\leq \kappa}$. It is enough to prove the following

Claim 1.1.c. For every $\gamma \in[\mu, \lambda)$ with $\kappa \geq \operatorname{cf}(\gamma)$, there is an $a \in \mathcal{P}$ such that $\gamma \in X_{a}^{\oplus}$.

Proof. It suffices to prove this for $\gamma \in[\mu, \lambda)$ with $\kappa \geq \operatorname{cf}(\gamma) \geq \aleph_{0}$. Let us define for such $\gamma$,

$$
\bar{\gamma}:= \begin{cases}\emptyset & \text { if } \operatorname{cf}(\gamma)=\aleph_{0}, \\ \left\{\gamma_{\varepsilon}: \varepsilon<\operatorname{cf}(\gamma)\right\} & \text { if } \operatorname{cf}(\gamma)>\aleph_{0},\end{cases}
$$

where $\left\{\gamma_{\varepsilon}: \varepsilon<\operatorname{cf}(\gamma)\right\}$ is an increasing enumeration of a club of $\gamma$. Let $\theta$ be large enough, so that $\mathfrak{A} \in H(\theta)$, and let $x=\langle\gamma, \bar{\gamma}, \mathfrak{A}\rangle$. Let also $\mathfrak{B}:=$ $\left\langle H(\theta), \in, \prec^{*}\right\rangle$.

Using Theorem $\mathrm{C}$, we get a sequence $\left\langle N_{i}: i<\kappa^{+}\right\rangle$and club $E$ of $\kappa^{+}$ which exemplify the theorem for our chosen $x$.

Now, for any $i$, we know that $N_{i} \cap \gamma \in N_{i+1}$. Hence, $\operatorname{cl}\left(N_{i} \cap \gamma\right) \cap \gamma \subseteq N_{i+1}$.

So, if $\operatorname{cf}(i)>\aleph_{0}$, then $N_{i} \cap \gamma$ is $\omega$-closed. Note that for any $i$, we have $\{\mathfrak{A}\} \cup\left(N_{i} \cap \mu\right) \cup\{\gamma\} \subseteq N_{i} \prec \mathfrak{B}$, so $\operatorname{Sk}_{\mathfrak{A}}\left(\left(N_{i} \cap \mu\right) \cup\{\gamma\}\right) \subseteq N_{i}$, hence $\operatorname{Sk}_{\mathfrak{A}}\left(\left(N_{i} \cap \mu\right) \cup\{\gamma\}\right) \cap \mu=N_{i} \cap \mu$.

So, if $\operatorname{cf}(\gamma)=\aleph_{0}$ and $i \in E$ is such that $\operatorname{cf}(i)>\aleph_{0}$, then $\gamma \in X_{N_{i} \cap \mu}$. Similarly, if $a=N_{i} \cap \mu$, then $Y_{a, \gamma}=N_{i} \cap \gamma \cdot$-1.1.c

The conclusion of the theorem follows easily, the sets with squares are $X_{a}^{\oplus}$ for $a \in \mathcal{P}$.

Remark 1.2. (0) Note that we have obtained an alternative proof that under the assumptions of 1.1 , we have $S_{\leq \kappa}^{\lambda} \in I[\lambda]$. This was proved in [Sh $420,2.8]$.

(1) Theorem 1.1 strengthens [Sh 237e, 2] for $\mu$ singular, as [Sh 237e, 2] had the same conclusion under $\mu^{\leq \kappa}=\mu$ instead of $\operatorname{cf}\left([\mu]^{\leq \kappa}, \subseteq\right)=\mu$. 
(2) If $\aleph_{0}<\operatorname{cf}(\kappa)=\kappa \leq \operatorname{cf}(\mu)<\mu$, what is the strength of the assumption $\operatorname{cf}([\mu] \leq \kappa, \subseteq)=\mu$ ? In [Sh 430, 1.3] it is proved that this follows from $\operatorname{pp}(\mu)=$ $\mu^{+}$. If $\operatorname{cf}\left([\mu]^{\aleph_{0}}, \subseteq\right)=\mu$ and for all $\theta \in(\kappa, \mu)$ we have $\theta>\operatorname{cf}(\theta)<\operatorname{cf}(\mu) \Rightarrow$ $\operatorname{pp}(\theta) \leq \mu$, then $\operatorname{cf}([\mu] \leq \kappa, \subseteq)=\mu$.

A particular situation in which our theorem applies and is not implied by the previously known theorems, is for example $\mu=\aleph_{\omega_{17}}$ and $\kappa=\aleph_{13}$ (see [Sh 400, Why the HELL is it four?]).

1.3. Acknowledgement. We would like to thank James Cummings for noticing a mistake in an earlier version of the theorem.

We shall now turn our attention to successors of singular strong limits, for which we can prove that a weak version of the square property holds. It will be useful to define the notion of a square pretender, as follows.

Definition 1.4. Suppose that $\kappa<\mu$ are cardinals, $\kappa$ is regular, and $e \subseteq \mu$. A square pretender on e of depth $\kappa$ is a sequence

$$
\left\langle S_{i}, \bar{d}^{i}=\left\langle d_{\gamma}^{i}: \gamma \in S_{i}\right\rangle, \bar{s}^{\gamma, i}=\left\langle\alpha_{\zeta}^{\gamma, i}: \zeta \in d_{\gamma}^{i}\right\rangle: i<\kappa\right\rangle
$$

such that:

(a) $\bigcup_{i<\kappa} S_{i} \supseteq\{\beta \in e: \operatorname{cf}(\beta) \neq \kappa\}$ and $\bigcup_{i<\kappa} \bigcup_{\gamma \in S_{i}}\left\{\alpha_{\zeta}^{\gamma, i}: \zeta \in d_{\gamma}^{i}\right\} \supseteq$ $\{\beta \in e: \operatorname{cf}(\beta) \neq \kappa\}$.

(b) $\left\langle\alpha_{\zeta}^{\gamma, i}: \zeta \in d_{\gamma}^{i}\right\rangle$ is an increasing sequence of elements of $\gamma$.

(c) If $\zeta \in d_{\gamma}^{i}$, then $\alpha_{\zeta}^{\gamma, i} \in S_{i}$ and $d_{\alpha_{\zeta}^{\gamma, i}}^{i} \subseteq d_{\gamma}^{i}$ and $\bar{s}_{\zeta}^{\alpha_{\zeta}^{\gamma, i}, i}=\left\langle\alpha_{\zeta^{\prime}}^{\gamma, i}: \zeta^{\prime} \in\right.$ $\left.d_{\alpha_{\zeta}^{\gamma, i}}^{i}\right\rangle$

Before we state the following theorem, we remind the reader of the following.

We shall be concerned with $\lambda=\mu^{+}$for $\mu$ a strong limit singular cardinal. By [Sh 108] or [Sh 88a] in this situation there is a maximal $W^{*}$ in $I[\lambda]$, which is unique up to a nonstationary set. In other words, for every $W \subseteq \lambda$, we have $W \in I[\lambda] \Leftrightarrow W \backslash W^{*} \in \mathrm{NS}[\lambda]$. (The reader unfamiliar with the ideal $I[\lambda]$ will find a lot about it in $\S 3$, including the meaning of $\left\langle C_{\delta}: \delta<\lambda\right\rangle$ witnessing that $W^{*} \in I[\lambda]$, which is needed for the statement of the following theorem.)

On to the theorem:

THEOREM 1.5. Suppose that $\mu$ is a strong limit singular cardinal of cofinality $\kappa$ and $\lambda=\mu^{+}$. Let $W^{*}$ be the maximal element of $I[\lambda]$ and let $\left\langle C_{\delta}: \delta<\lambda\right\rangle$ witness that $W^{*} \in I[\lambda]$. Let $E=\operatorname{acc}\left(E_{0}\right)$, where $E_{0}$ is a club of $\lambda$ such that for every $\delta \in W^{*} \cap E_{0}, C_{\delta}$ is a club of $\delta$ of order type $\operatorname{cf}(\delta)<\delta($ see 3.0(2)). Then there is a sequence

$$
\left\langle S_{j}, \bar{d}^{j}=\left\langle d_{\gamma}^{j}: \gamma \in S_{j}\right\rangle, \bar{s}^{\gamma, j}=\left\langle\alpha_{\zeta}^{\gamma, j}: \zeta \in d_{\gamma}^{j}\right\rangle: j<\mu\right\rangle
$$


such that for every $\delta \in W^{*} \cap E$ and for every club $e \subseteq \operatorname{acc}\left(C_{\delta}\right)$, there is a sequence $\left\langle j_{i}: i<\kappa\right\rangle$ in $\mu$ such that $\left\langle S_{j_{i}}, \bar{d}^{j_{i}}, \bar{s}^{\gamma, j_{i}}: i<\kappa\right\rangle$ is a square pretender on $e$ of depth $\kappa$.

Proof. Let us fix an increasing sequence $\left\langle\mu_{i}: i<\kappa\right\rangle$ of cardinals such that $\mu=\sum_{i<\kappa} \mu_{i}$. We also choose, by induction on $\alpha<\lambda$, sets $a_{i}^{\alpha}$ for $i<\kappa$ with the following properties:

(a) $\alpha=\bigcup_{i<\kappa} a_{i}^{\alpha}$.

(b) $\left|a_{i}^{\alpha}\right| \leq \mu_{i}$.

(c) $\alpha \in a_{i}^{\beta} \Rightarrow a_{i}^{\alpha} \subseteq a_{i}^{\beta}$.

(d) $i<j<\kappa \Rightarrow a_{i}^{\alpha} \subseteq a_{j}^{\alpha}$.

(e) $\left|C_{\alpha}\right| \leq \mu_{i} \Rightarrow C_{\alpha} \subseteq a_{i}^{\alpha}$.

Let us also define for $\alpha<\beta<\lambda$,

$$
c(\alpha, \beta):=\min \left\{i<\kappa: \alpha \in a_{i}^{\beta}\right\} ;
$$

so $\alpha<\beta<\gamma \Rightarrow c(\alpha, \gamma) \leq \max \{c(\alpha, \beta), c(\beta, \gamma)\}$, by (c) above.

Now let us fix a $\delta \in W^{*} \cap E$ and let $\theta=\operatorname{cf}(\delta)$; so $\theta$ is a regular cardinal $<\mu$. Suppose that $e \subseteq \operatorname{acc}\left(C_{\delta}\right)$ is a fixed club of $\delta$. Therefore otp $(e)=\theta$ (since $\left.\operatorname{otp}\left(C_{\delta}\right)=\operatorname{cf}(\delta)\right)$. We define for all $i<\kappa$ such that $\mu_{i} \geq \theta$,

$$
A_{\delta, i}=A_{\delta, i, e}:=\left\{\alpha \in e: a_{i}^{\alpha} \cap \operatorname{nacc}\left(C_{\delta}\right) \text { is unbounded in } \alpha\right\} .
$$

If $\mu_{i}<\theta$, we define $A_{\delta, i}=A_{\delta, i, e}:=\emptyset$.

We prove some facts about the just defined sets, which will prepare the ground for further definitions.

FACT 1.5.a. If $\alpha_{1}<\alpha_{2} \in A_{\delta, i}$, then $a_{i}^{\alpha_{1}}$ is a bounded subset of $a_{i}^{\alpha_{2}}$ (hence $\operatorname{otp}\left(a_{i}^{\alpha_{1}}\right)<\operatorname{otp}\left(a_{i}^{\alpha_{2}}\right)$ ).

Proof. Since $\alpha_{2} \in A_{\delta, i}$, we can find a $\beta \in \operatorname{nacc}\left(C_{\delta}\right) \cap a_{i}^{\alpha_{2}}$ which is $>\alpha_{1}$. By $\beta \in \operatorname{nacc}\left(C_{\delta}\right)$, we have $C_{\beta}=C_{\delta} \cap \beta$ (see 3.0(2)). Now, since $\alpha_{1} \in e$, in particular $\alpha_{1} \in C_{\delta}$, so $\alpha_{1} \in C_{\beta}$. By (e) and (c) we have $a_{i}^{\alpha_{1}} \subseteq a_{i}^{\beta}$. But $a_{i}^{\beta} \subseteq a_{i}^{\alpha_{2}}$ as $\beta \in a_{i}^{\alpha_{2}}$. Obviously, by $\alpha_{2} \in A_{\delta, i}$, we have $\sup \left(a_{i}^{\alpha_{2}}\right)=\alpha_{2}>$ $\alpha_{1} \geq \sup \left(a_{i}^{\alpha_{1}}\right)$.

FACT 1.5.b. $\left\langle A_{\delta, i}: i<\kappa\right\rangle$ is an increasing sequence of subsets of $e$.

Proof. This follows, since $a_{i}^{\alpha}$ are increasing.

FACT 1.5.c. $\bigcup_{i<\kappa} A_{\delta, i} \supseteq\{\gamma \in e: \operatorname{cf}(\gamma) \neq \kappa\}$.

Proof. For any $\gamma \in e \subseteq \operatorname{acc}\left(C_{\delta}\right)$, we have $\gamma=\sup \left(C_{\delta} \cap \gamma\right)=\sup \left(\operatorname{nacc}\left(C_{\delta}\right)\right.$ $\cap \gamma)$, so $\gamma=\sup \left(\operatorname{nacc}\left(C_{\delta}\right) \cap \bigcup_{i<\kappa} a_{i}^{\gamma}\right)$. If $\operatorname{cf}(\gamma) \neq \kappa$, then there is an $i<\kappa$ such that $\gamma=\sup \left(\operatorname{nacc}\left(C_{\delta}\right) \cap a_{i}^{\gamma}\right)$. As $a_{i}^{\gamma}$ are increasing with $i$, there is an $i$ such that $\mu_{i} \geq \theta$. $1.5 . c$ 
Remark 1.5.d (this remark is not used later in the proof). Suppose $e \subseteq E_{0}$. If $\gamma \in e$ is such that $A_{\delta, i} \cap \gamma$ is stationary in $\gamma$ and $\left|C_{\gamma}\right| \leq \mu_{i}$, then $\gamma \in A_{\delta, i}$.

Proof. Since $\gamma \in e \subseteq \operatorname{acc}\left(C_{\delta}\right)$, the set $T:=A_{\delta, i} \cap C_{\delta} \cap C_{\gamma}$ is stationary in $\gamma$ (as $\gamma \in E_{0}$ ). If $\beta \in T$, by (e) in the definition of $a$ 's, we have $a_{i}^{\beta} \subseteq a_{i}^{\gamma}$. Therefore

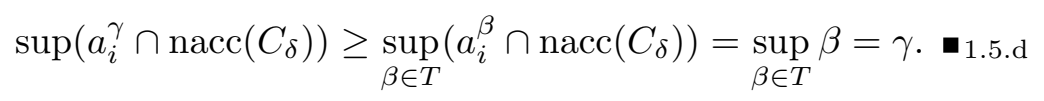

Continuation of the proof of 1.5. Let us now fix an $i(*)<\kappa$. We enumerate $A_{\delta, i(*)}$ increasingly as

$$
A_{\delta, i(*)}=\left\{\alpha_{\varepsilon}^{\delta}: \varepsilon<\operatorname{otp}\left(A_{\delta, i(*)}\right) \leq \theta\right\},
$$

and set $\alpha_{\theta}^{\delta}=\delta$. For $\varepsilon \leq \theta$ for which $\alpha_{\varepsilon}^{\delta}$ is defined, we put

$$
d_{\varepsilon}^{i(*)}:=\left\{\zeta<\varepsilon: c\left(\alpha_{\zeta}^{\delta}, \alpha_{\varepsilon}^{\delta}\right) \leq i(*)\right\}
$$

and for $\zeta \leq \varepsilon \leq \theta$,

$$
b_{\zeta, \varepsilon}^{i(*)}:=\operatorname{otp}\left(\alpha_{\zeta}^{\delta} \cap a_{i(*)}^{\alpha_{\varepsilon}^{\delta}}\right) .
$$

We define a partial function $\varepsilon: \lambda \rightarrow \theta$ by setting $\varepsilon(\alpha)$ to be the (unique by 1.5.f below) $\varepsilon$ such that we can find a sequence $\left\langle\alpha_{\zeta}: \zeta \in d_{\varepsilon}^{i(*)}\right\rangle$ in $\alpha$ with the following properties:

(A) $\left\langle\alpha_{\zeta}: \zeta \in d_{\varepsilon}^{i(*)}\right\rangle$ increases with $\zeta$.

(B) $c\left(\alpha_{\zeta}, \alpha\right) \leq i(*)$.

(C) $\operatorname{otp}\left(\alpha_{\zeta} \cap a_{i(*)}^{\alpha}\right)=b_{\zeta, \varepsilon}^{i(*)}$.

(D) If $\zeta_{1}<\zeta_{2}$ are in $d_{\varepsilon}^{i(*)}$ and $c\left(\alpha_{\zeta_{1}}^{\delta}, \alpha_{\zeta_{2}}^{\delta}\right) \leq i(*)$, then

$$
c\left(\alpha_{\zeta_{1}}, \alpha_{\zeta_{2}}\right)=c\left(\alpha_{\zeta_{1}}^{\delta}, \alpha_{\zeta_{2}}^{\delta}\right) \quad \text { and } \quad \operatorname{otp}\left(\alpha_{\zeta_{1}} \cap a_{i(*)}^{\alpha_{\zeta_{2}}}\right)=\operatorname{otp}\left(\alpha_{\zeta_{1}}^{\delta} \cap a_{i(*)}^{\alpha_{\zeta_{2}}^{\delta}}\right) \text {. }
$$

(E) $\operatorname{otp}\left(a_{i(*)}^{\alpha}\right)=\operatorname{otp}\left(a_{i(*)}^{\alpha_{\varepsilon}^{\delta}}\right)$ by an order preserving isomorphism which exemplifies that $\operatorname{otp}\left(a_{i(*)}^{\alpha_{\zeta}}\right)=\operatorname{otp}\left(a_{i(*)}^{\alpha_{\zeta}^{\delta}}\right)$ for all $\zeta \in d_{\varepsilon}^{i(*)}$.

We prove several facts about the partial function $\varepsilon$ and sets $d_{\varepsilon}^{i(*)}$.

FACT 1.5.e. If $\varepsilon_{1} \in d_{\varepsilon}^{i(*)}$, then $d_{\varepsilon_{1}}^{i(*)} \subseteq d_{\varepsilon}^{i(*)}$.

Proof. If $\zeta \in d_{\varepsilon_{1}}^{i(*)}$, then $\zeta<\varepsilon_{1}$ and $c\left(\alpha_{\zeta}^{\delta}, \alpha_{\varepsilon_{1}}^{\delta}\right) \leq i(*)$. Since $\varepsilon_{1} \in d_{\varepsilon}^{i(*)}$, also $\varepsilon_{1}<\varepsilon$ and $c\left(\alpha_{\varepsilon_{1}}^{\delta}, \alpha_{\varepsilon}^{\delta}\right) \leq i(*)$. So $\zeta<\varepsilon$ and $c\left(\alpha_{\zeta}^{\delta}, \alpha_{\varepsilon}^{\delta}\right) \leq i(*)$. 1.5.e

FACT 1.5.f. If $\alpha<\lambda$, there is at most one $\varepsilon$ and $\left\langle\alpha_{\zeta}: \zeta \in d_{\varepsilon}^{i(*)}\right\rangle$ which satisfy (A)-(E). 
Proof. Suppose first that $\left\langle\alpha_{\zeta}: \zeta \in d_{\varepsilon}^{i(*)}\right\rangle$ and $\left\langle\alpha_{\zeta}^{\prime}: \zeta \in d_{\varepsilon}^{i(*)}\right\rangle$ both exemplify $(\mathrm{A})-(\mathrm{E})$. Then for each $\zeta \in d_{\varepsilon}^{i(*)}$, by $(\mathrm{B})$,

$$
\alpha_{\zeta}, \alpha_{\zeta}^{\prime} \in a_{i(*)}^{\alpha} .
$$

By $(\mathrm{C})$,

$$
\operatorname{otp}\left(\alpha_{\zeta} \cap a_{i(*)}^{\alpha}\right)=\operatorname{otp}\left(\alpha_{\zeta}^{\prime} \cap a_{i(*)}^{\alpha}\right),
$$

so it must be that $\alpha_{\zeta}=\alpha_{\zeta}^{\prime}$, for all $\zeta \in d_{\varepsilon}^{i(*)}$.

Suppose then that $\left\langle\alpha_{\zeta}: \zeta \in d_{\varepsilon_{1}}^{i(*)}\right\rangle$ and $\left\langle\beta_{\zeta}: \zeta \in d_{\varepsilon_{2}}^{i(*)}\right\rangle$ both exemplify (A)-(E) for $\varepsilon_{1}<\varepsilon_{2}$. By (E),

$$
\operatorname{otp}\left(a_{i(*)}^{\alpha_{\varepsilon_{1}}^{\delta}}\right)=\operatorname{otp}\left(a_{i(*)}^{\alpha}\right)=\operatorname{otp}\left(a_{i(*)}^{\alpha_{\varepsilon_{2}}^{\delta}}\right),
$$

which is a contradiction with $\alpha_{\varepsilon_{1}}^{\delta}<\alpha_{\varepsilon_{2}}^{\delta} \in A_{\delta, i(*)}$, by 1.5.a.

FACT 1.5.g. If $\varepsilon(\alpha)$ is well defined, and witnessed by $\left\langle\alpha_{\zeta}: \zeta \in d_{\varepsilon(\alpha)}^{i(*)}\right\rangle$, then, for every $\zeta \in d_{\varepsilon(\alpha)}^{i(*)}$, we have $\varepsilon\left(\alpha_{\zeta}\right)=\zeta$ and this is witnessed by $\left\langle\alpha_{\zeta^{\prime}}: \zeta^{\prime} \in d_{\zeta}^{i(*)}\right\rangle$.

Proof. By Fact 1.5.e, we have $d_{\zeta}^{i(*)} \subseteq d_{\varepsilon(\alpha)}^{i(*)}$, so $\left\langle\alpha_{\zeta^{\prime}}: \zeta^{\prime} \in d_{\zeta}^{i(*)}\right\rangle$ is well defined. We need to check that $(\mathrm{A})-(\mathrm{E})$ are satisfied.

(A) is obviously true, so consider (B). By (D) for $\varepsilon(\alpha)$ and the definition of $d_{\zeta}^{i(*)}$, if $\zeta^{\prime} \in d_{\zeta}^{i(*)}$, then $c\left(\alpha_{\zeta^{\prime}}, \alpha_{\zeta}\right)=c\left(\alpha_{\zeta^{\prime}}^{\delta}, \alpha_{\zeta}^{\delta}\right) \leq i(*)$.

To see (C), use again (D), so otp $\left(\alpha_{\zeta^{\prime}} \cap a_{i(*)}^{\alpha_{\zeta}}\right)=\operatorname{otp}\left(\alpha_{\zeta^{\prime}}^{\delta} \cap a_{i(*)}^{\alpha_{\zeta}^{\delta}}\right)$, which is by definition equal to $b_{\zeta^{\prime}, \zeta}^{i(*)}$.

Now, (D) follows from (D) for $\varepsilon(\alpha)$, and the fact that $d_{\zeta}^{i(*)} \subseteq d_{\varepsilon(\alpha)}^{i(*)}$. The last statement also implies $(E)$.

Continuation of the proof of 1.5. Now we can set

$$
S_{i(*)}=S_{\delta, i(*), e}:=\{\alpha \in \lambda: \varepsilon(\alpha) \text { is well defined }\} .
$$

Note that $S_{i(*)} \supseteq A_{\delta, i(*)}$, as for $\alpha=\alpha_{\varepsilon(*)}^{\delta}$ we have $\varepsilon(\alpha)=\varepsilon(*)$. Also note that $\bigcup_{i<\kappa} \bigcup_{\alpha \in S_{i}}\left\{\alpha_{\zeta}: \zeta \in d_{\varepsilon(\alpha)}^{i}\right\} \supseteq \bigcup_{i<\kappa} A_{\delta, i}$.

Therefore $\left\langle S_{i},\left\langle d_{\varepsilon(\alpha)}^{i}: \alpha \in S_{i}\right\rangle,\left\langle\alpha_{\zeta}: \zeta \in d_{\varepsilon(\alpha)}^{i}\right\rangle: i\langle\kappa\rangle\right.$ is a square pretender of depth $\kappa$ on $e$.

Now notice that the choice of this square pretender only depended on the following parameters:

(i) $\theta<\mu$.

(ii) $\left\langle\operatorname{otp}\left(A_{\delta, i}\right): i<\kappa\right\rangle$, which is a $\kappa$-sequence of elements of $\theta+1$.

(iii) $\left\langle\left\langle d_{\varepsilon}^{i}: \varepsilon \leq \theta\right\rangle: i<\kappa\right\rangle$, and each $d_{\varepsilon}^{i}$ is a subset of $\varepsilon$.

(iv) $\left\langle\left\langle b_{\zeta, \varepsilon}^{i}: \zeta \leq \varepsilon \leq \theta\right\rangle: i<\kappa\right\rangle$ and each $b_{\zeta, \varepsilon}^{i}$ is an element of $\mu_{i}^{+}$. 
By the fact that $\mu$ is a strong limit, all possible such choices can be enumerated in type $\mu$, so the theorem is proved.

2. On singular ex-inaccessible and outside guessing of clubs. In this section we consider the following question. Suppose we start with two universes $V_{1} \subseteq V_{2}$ of set theory and a cardinal $\kappa$ which is inaccessible in $V_{1}$. We assume $\kappa$ is singular in $V_{2}$, and then want to "guess" clubs of $\kappa$ from $V_{1}$ (we refer to this as to "outside guessing of clubs"). The history of this question and related results of Shelah, and Gitik, were presented in the introduction.

We have two ways of "guessing": the first one is to find an unbounded subset (or, equivalently, find a club) of $\kappa$ in $V_{2}$ which is almost contained in every club of $\kappa$ in $V_{1}$. The other "guessing" is by a proper filter. To obtain these guessings we need additional assumptions, which go in two directions. One is the cardinal arithmetic in $V_{1}$. If the extension $V_{2}$ was obtained through forcing, the other set of restrictions can be regarded as speaking on the chain condition of the forcing used. In fact, these restrictions are about certain covering numbers.

Throughout this section, if we are simultaneously speaking of two universes of set theory, $V_{1}$ and $V_{2}$, such that $V_{1} \subseteq V_{2}$, and we have not specified in which one we carry the argument, then we mean $V_{2}$. The symbol $\Delta$ stands for the diagonal intersection.

We now proceed to the results.

Theorem 2.0. Assume that:

(i) $V_{1} \subseteq V_{2}$ are transitive classes containing the ordinals and modeling $Z F C$.

(ii) $V_{1} \vDash " \kappa$ is inaccessible".

(iii) $V_{2} \vDash " \kappa$ is a singular cardinal, $\operatorname{cf}(\kappa)=\theta$ and $\kappa^{+}=\left(\kappa^{+}\right)^{V_{1}}$ ".

(iv) $V_{1} \vDash " \operatorname{cf}(\operatorname{Club}(\kappa), \supseteq) \leq \kappa^{+} "$.

Then:

(1) In $V_{2}$, we can find an unbounded $C^{*} \subseteq \kappa$ such that:

$$
E \in \operatorname{Club}^{V_{1}}(\kappa) \Rightarrow C^{*} \backslash E \text { is bounded. }
$$

(2) If $\gamma<\kappa$ is fixed, we can also demand that

$$
\beta \in \operatorname{nacc}\left(C^{*}\right) \Rightarrow \operatorname{cf}^{V_{2}}(\beta)>\gamma .
$$

(3) If $V_{2} \vDash " \theta>\aleph_{0} "$ ", we can extend (1):

$$
\alpha \in \operatorname{nacc}\left(C^{*}\right) \Rightarrow \operatorname{cf}^{V_{2}}(\alpha) \geq \sup \left(C^{*} \cap \alpha\right) .
$$

Proof. (1) In $V_{1}$, by [Sh 351, §4] $=$ [Sh 365, 2.14], we can find sets $S_{i}$ $(i<\kappa)$ such that: 
$(\alpha) \bigcup_{i<\kappa} S_{i}=\left\{\delta<\kappa^{+}: \operatorname{cf}(\delta)<\kappa\right\}$.

( $\beta$ ) For each $i<\kappa$, there is a square $\left\langle C_{\alpha}^{i}: \alpha \in S_{i}\right\rangle$ on $S_{i}$ (see 1.0 for the definition).

In addition to $(\alpha)$ and $(\beta)$, since $\kappa$ is a limit cardinal (again by [Sh 351, $\S 4]=[$ Sh 365, 2.14]), we can assume that

$(\gamma)$ For each $i<\kappa$ there is a $\mu_{i}<\kappa$ such that for all $\alpha \in S_{i}$ we have $\operatorname{otp}\left(C_{\alpha}^{i}\right)<\mu_{i}$.

Since $\left(\kappa^{+}\right)^{V_{1}}=\left(\kappa^{+}\right)^{V_{2}}$, and it is easily seen that $(\beta)$ and $(\gamma)$ are absolute between $V_{1}$ and $V_{2}$, the sets $S_{i}(i<\kappa)$ will still satisfy $(\beta)-(\gamma)$ in $V_{2}$, and, by $(\alpha)$, we shall have $\bigcup_{i<\kappa} S_{i} \supseteq\left\{\delta<\kappa^{+}: \operatorname{cf}^{V_{2}}(\delta) \neq \theta\right\}$.

Now we argue in $V_{2}$. We fix a regular $\mu^{*} \in(\theta, \kappa)$.

Since $S_{\mu^{*}}^{\kappa^{+}}:=\left\{\alpha<\kappa^{+}: \mathrm{cf}^{V_{2}}(\alpha)=\mu^{*}\right\}$ is stationary in $\kappa^{+}$and included in $\bigcup_{i<\kappa} S_{i}$, we can fix an $i(*)<\kappa$ such that $S_{i(*)} \cap S_{\mu^{*}}^{\kappa^{+}}$is stationary. If we take any club $C$ of $\kappa^{+}$which is in $V_{2}$, and if $\alpha \in S_{i(*)} \cap S_{\mu^{*}}^{\kappa^{+}} \cap \operatorname{acc}(C)$, then $\operatorname{cf}^{V_{2}}(\alpha)=\mu^{*}$ and $C \cap C_{\alpha}^{i(*)}$ is a club of $\alpha$. To summarize, we can conclude that

$(\delta)$ There is an $i(*)<\kappa$ such that, in $V_{2}$, for every club $C \in V_{2}$ of $\kappa^{+}$, for stationarily many $\alpha \in S_{i(*)}$,

$$
C_{\alpha}^{i(*)} \cap C \text { is a club of } \alpha \& \operatorname{cf}^{V_{2}}(\alpha)=\mu^{*} .
$$

OBSERVATION. In $V_{1}$, by $\mathrm{cf}^{V_{1}}(\mathrm{Club}(\kappa), \supseteq) \leq \kappa^{+}$we can fix a sequence $\left\langle E_{\alpha}: \alpha<\kappa^{+}\right\rangle \in V_{1}$ of clubs in $\kappa$ such that:

(i) For every $E \in \operatorname{Club}^{V_{1}}(\kappa)$, for some $\alpha$ we have $E \supseteq E_{\alpha}$.

(ii) $\beta<\alpha \Rightarrow E_{\beta} \supseteq{ }^{*} E_{\alpha}$.

(iii) $\beta \in C_{\alpha}^{i(*)} \Rightarrow E_{\beta} \supseteq E_{\alpha}$.

[Why? This can be done by induction: suppose that $\left\langle D_{\alpha}: \alpha<\kappa^{+}\right\rangle \in V_{1}$ is a sequence of clubs of $\kappa$ which is cofinal in $\left(\operatorname{Club}^{V_{1}}(\kappa), \supseteq\right)$. Let $E_{0}=D_{0}$, and suppose that $\left\langle E_{\beta}: \beta<\alpha\right\rangle$ are given for an $\alpha<\kappa^{+}$. Note that the following is well defined:

$$
\gamma_{\alpha}:=\min \left\{\gamma:(\forall \beta<\alpha)\left(D_{\gamma} \subseteq^{*} E_{\beta}\right)\right\} .
$$

Namely, $E^{\beta}:=\Delta_{\beta<\alpha} E_{\beta} \in \operatorname{Club}^{V_{1}}(\kappa)$ and $(\forall \beta<\alpha)\left(E^{\beta} \subseteq^{*} E_{\beta}\right)$. So, there is some $\gamma$ such that $D_{\gamma} \subseteq^{*} E^{\beta}$.

Now, if $\gamma_{\alpha} \in S_{i(*)}$, let

$$
E_{\alpha}:=D_{\alpha} \cap D_{\gamma_{\alpha}} \cap \bigcap_{\beta \in C_{\alpha}^{i(*)}} E_{\beta} .
$$

If $\alpha \notin S_{i(*)}$, we simply skip the last term in the above intersection. Note that we are using $(\gamma)$ above to assure that each $E_{\alpha}$ is a club.] 
The rest of the argument takes place in $V_{2}$. We let $\left\langle\xi_{\varepsilon}: \varepsilon<\theta\right\rangle$ be a strictly increasing cofinal sequence in $\kappa$, and if $\theta>\aleph_{0}$ we also require that this sequence is continuous. In addition, we require that $\xi_{\varepsilon+1}$ is a successor ordinal, for every $\varepsilon<\theta$.

For every $\alpha \in \kappa^{+}$, we define

$$
c_{\alpha}:=\left\{\varepsilon<\theta:\left(\xi_{\varepsilon}, \xi_{\varepsilon+1}\right) \cap E_{\alpha} \neq \emptyset\right\}, \quad d_{\alpha}:=\left\{\sup \left(\xi_{\varepsilon+1} \cap E_{\alpha}\right): \varepsilon \in c_{\alpha}\right\} .
$$

Note that $d_{\alpha} \subseteq E_{\alpha} \backslash\left\{\xi_{\varepsilon}: \varepsilon<\theta\right\}$ and $\sup \left(d_{\alpha}\right)=\kappa$, for each $\alpha<\kappa^{+}$.

Now we can distinguish two cases:

Case (A): For some $\alpha$, for every $\beta \in\left(\alpha, \kappa^{+}\right)$, the symmetric difference $d_{\beta} \triangle d_{\alpha}$ is bounded in $\kappa$. We set $C^{*}=d_{\alpha}$ for some such $\alpha$, and easily check that (1) holds.

Case (B): Not (A). Therefore, for every $\alpha \in \kappa^{+}$, there is a minimal $f(\alpha) \in\left(\alpha, \kappa^{+}\right)$such that $\kappa=\sup \left(d_{\alpha} \triangle d_{f(\alpha)}\right)$. The following can be noticed:

OBSERVATION. We have

$$
f(\alpha) \leq \beta<\kappa \Rightarrow \kappa=\sup \left(d_{\alpha} \triangle d_{\beta}\right) .
$$

[Why? If $\gamma$ is such that $\left(E_{f(\alpha)} \backslash E_{\alpha}\right) \cup\left(E_{\beta} \backslash E_{f(\alpha)}\right) \subseteq \gamma$, and $\varepsilon \in c_{\beta} \cap c_{\alpha}$ is such that $\sup \left(E_{\beta} \cap \xi_{\varepsilon+1}\right)=\sup \left(E_{\alpha} \cap \xi_{\varepsilon+1}\right)$ and $\xi_{\varepsilon} \geq \gamma$, then we also have $\sup \left(E_{\alpha} \cap \xi_{\varepsilon+1}\right)=\sup \left(E_{f(\alpha)} \cap \xi_{\varepsilon+1}\right)$.]

Let

$$
E^{*}:=\left\{\delta<\kappa^{+}: \delta \text { is a limit ordinal such that } \bigwedge_{\alpha<\delta} f(\alpha)<\delta\right\} .
$$

Obviously, $E^{*} \in V_{2}$ is a club of $\kappa^{+}$. Then by $(\delta)$, for some $\alpha^{*} \in S_{i(*)}$ we have

$$
V_{2} \vDash " \operatorname{cf}\left(\alpha^{*}\right)=\mu^{*} \& C_{\alpha^{*}}^{i(*)} \cap E^{*} \text { is a club of } \alpha^{*} " .
$$

Let $\left\langle\beta_{i}: i<\varrho\right\rangle$ be an increasing enumeration of $\operatorname{acc}\left(C_{\alpha^{*}}^{i(*)} \cap E^{*}\right)$, for some $\varrho \geq \mu^{*}$. We construct by induction on $i$ an increasing subsequence $\left\langle\xi_{\varepsilon_{i}}: i<\mu^{*}\right\rangle$ of $\left\langle\xi_{\varepsilon}: \varepsilon<\theta\right\rangle$, thus obtaining a contradiction with $\mu^{*}>\theta$.

Let us introduce the notation $\delta_{\varepsilon}^{\alpha}:=\sup \left(\xi_{\varepsilon+1} \cap E_{\alpha}\right)$ for $\varepsilon \in c_{\alpha}$ and $\alpha \in \kappa^{+}$.

We first make two observations:

$\left(\mathrm{o}_{1}\right)$ If $i<j<\varrho$, then by the square property of $C_{\alpha^{*}}^{i(*)}$ and (iii) above, $E_{\beta_{i}} \supseteq E_{\beta_{j}}$. So, if $i<j<k<\varrho$ and $\varepsilon<\theta$ is such that $\left(d_{\beta_{i}} \triangle d_{\beta_{j}}\right) \cap$ $\left(\xi_{\varepsilon}, \xi_{\varepsilon+1}\right) \neq \emptyset$ and $\left(d_{\beta_{j}} \triangle d_{\beta_{k}}\right) \cap\left(\xi_{\varepsilon}, \xi_{\varepsilon+1}\right) \neq \emptyset$, then $\delta_{\varepsilon}^{\beta_{i}}>\delta_{\varepsilon}^{\beta_{j}}>\delta_{\varepsilon}^{\beta_{k}}$.

$\left(\mathrm{o}_{2}\right)$ Given $i, j<\varrho$ (so $f\left(\beta_{i}\right)<\beta_{j}$ ), and $\varepsilon<\theta$, there is a $\zeta>\varepsilon$ such that $\left(d_{\beta_{i}} \triangle d_{\beta_{j}}\right) \cap\left(\xi_{\zeta}, \xi_{\zeta+1}\right) \neq \emptyset$. This follows from the $(*)$ above.

Now we proceed to construct $\left\langle\xi_{\varepsilon_{i}}: i<\mu^{*}\right\rangle$. 
Let $\gamma_{0}^{0}=\beta_{0}$ and $\gamma_{1}^{0}=\beta_{1}$ (so $\left.\gamma_{1}^{0}>f\left(\gamma_{0}^{0}\right)\right)$ and let $\varepsilon_{0}$ be such that $\left(d_{\gamma_{0}^{0}} \triangle d_{\gamma_{1}^{0}}\right) \cap\left(\xi_{\varepsilon_{0}}, \xi_{\varepsilon_{0}+1}\right) \neq \emptyset$.

Suppose that for some $i<\mu^{*}$, we have chosen $\gamma_{0}^{i}, \gamma_{1}^{i}$ and $\varepsilon_{i}$ so that $\left(d_{\gamma_{0}^{i}} \triangle d_{\gamma_{1}^{i}}\right) \cap\left(\xi_{\varepsilon_{i}}, \xi_{\varepsilon_{i}+1}\right) \neq \emptyset$. We wish to define $\varepsilon_{i+1}$. Using the chosen $\gamma_{0}^{i}, \gamma_{1}^{i}$, we build an increasing subsequence $\gamma_{0}^{i}, \gamma_{1}^{i}, \gamma_{2}^{i} \ldots$ of $\left\langle\beta_{k}: k<\varrho\right\rangle$ such that

$$
l_{1}<l_{2} \Rightarrow\left(\xi_{\varepsilon_{i}}, \xi_{\varepsilon_{i}+1}\right) \cap\left(d_{\gamma_{l_{1}}^{i}} \triangle d_{\gamma_{l_{2}}^{i}}\right) \neq \emptyset .
$$

By $\left(\mathrm{o}_{1}\right)$ above, the sequence $\gamma_{0}^{i}, \gamma_{1}^{i}, \gamma_{2}^{i} \ldots$ must stop after a finite number of stages, since otherwise we obtain an infinite decreasing sequence of ordinals. Let $\gamma_{1}^{i+1}>\gamma_{0}^{i+1}>\max \left\{\gamma_{l}^{i}: \gamma_{l}^{i}\right.$ is well defined $\}$ be such that $\gamma_{0}^{i+1}=\beta_{k_{0}}$ and $\gamma_{1}^{i+1}=\beta_{k_{1}}$ for some $k_{0}<k_{1}<\varrho$. Let $\varepsilon_{i+1}>\varepsilon_{i}$ be such that $\left(d_{\gamma_{0}^{i+1}} \backslash d_{\gamma_{1}^{i+1}}\right) \cap$ $\left(\xi_{\varepsilon_{i+1}}, \xi_{\varepsilon_{i+1}+1}\right) \neq \emptyset$. Such an $\varepsilon_{i+1}$ exists by $\left(\mathrm{o}_{2}\right)$ above.

If $i$ is a limit ordinal $<\mu^{*}$, we define $\gamma_{0}^{i}>\sup \left\{\gamma_{l}^{j}: j<i \& \gamma_{l}^{j}\right.$ is well defined $\}, \gamma_{0}^{i}=\beta_{k}$ for some $k<\varrho$, which is possible by the regularity of $\mu^{*}>\aleph_{0}$. We define $\gamma_{1}^{i}$ and $\varepsilon_{i}$ as above.

(2) Let $\gamma<\kappa$ be fixed. In $V_{2}$, let $\sigma$ be regular, $\sigma \in(\gamma, \kappa)$, so $\sigma$ is also regular in $V_{1}$. We follow the ideas of [Sh 365, §2] but the following is self-sufficient.

The plan is to replace in the proof of (1) each $d_{\alpha}$ by a somewhat larger set $d_{\alpha}^{\dagger}$, so that

$$
\beta \in \operatorname{nacc}\left(d_{\alpha}^{\dagger}\right) \Rightarrow \mathrm{cf}^{V_{2}}(\beta) \geq \sigma .
$$

In the proof of (1), $C^{*}=d_{\beta}$ for some $\beta$. The definition of $d_{\alpha}^{\dagger}$ 's will be such that putting $C^{*}=d_{\beta}^{\dagger}$ for the same $\beta$, the newly defined $C^{*}$ will still satisfy (1).

First, we shall enlarge each $d_{\alpha}$ to a $d_{\alpha}^{\dagger}$ so that

$$
\beta \in \operatorname{nacc}\left(d_{\alpha}^{\dagger}\right) \& \operatorname{cf}^{V_{2}}(\beta)<\sigma \Rightarrow \beta \in \operatorname{nacc}\left(E_{\alpha}\right) .
$$

In $V_{1}$, we choose for each $\delta \in \kappa$ a club $e_{\delta}$ in $\delta$ such that $\operatorname{otp}\left(e_{\delta}\right)=\operatorname{cf}^{V_{1}}(\delta)$, and define $\bar{e}:=\left\langle e_{\delta}: \delta<\kappa\right\rangle \in V_{1}$.

Fix an $\alpha<\kappa^{+}$and set $C:=\left\{\xi_{\varepsilon+1}: \varepsilon \in c_{\alpha}\right\}, E:=E_{\alpha}, d:=d_{\alpha}$. Then, in the notation of [Sh 365, §2], using the "glue" operator gl,

$$
\begin{gathered}
d=\operatorname{gl}(C, E):=\mathrm{gl}^{0}(C, E):=\mathrm{gl}_{\sigma, 0}^{1}(C, E, \bar{e}) \\
\quad:=\{\sup (\beta \cap E): \beta \in C, \beta>\min (E)\} .
\end{gathered}
$$

We shall have

$$
d_{\alpha}^{\dagger}=\mathrm{gl}_{\sigma}^{1}(C, E, \bar{e}):=\bigcup_{n \in \omega} \mathrm{gl}_{\sigma, n}^{1}(C, E, \bar{e}),
$$

where $\mathrm{gl}_{\sigma, n}^{1}$ are defined inductively on $n$ as follows $\left(\mathrm{gl}_{\sigma, 0}^{1}(C, E, \bar{e})\right.$ is given above): 


$$
\begin{aligned}
\mathrm{gl}_{\sigma, n+1}^{1}(C, E, \bar{e}):= & \mathrm{gl}_{\sigma, n}^{1}(C, E, \bar{e}) \cup\{\sup (\delta \cap E): \\
& \text { for some } \beta \in \operatorname{nacc}\left(\mathrm{gl}_{\sigma, n}^{1}(C, E, \bar{e})\right) \text { we have } \\
& \left.\operatorname{cf}^{V_{2}}(\beta)<\sigma \& \delta \in e_{\beta} \& \delta>\sup \left(E \cap \delta \cap \mathrm{gl}_{\sigma, n}^{1}(C, E, \bar{e})\right)\right\} .
\end{aligned}
$$

We can easily check that this definition leaves us in the situation of $(* *)$.

On the other hand, since $E \in V_{1}$ is a club of $\kappa>\sigma$, it follows that

$$
E^{\dagger}:=\{\delta \in E: \operatorname{otp}(\delta \cap E) \text { is divisible by } \sigma\}
$$

is a club of $\kappa$, is in $V_{1}$ (because $E \in V_{1}$ ), and has the property that

$$
\beta \in \operatorname{nacc}\left(E^{\dagger}\right) \Rightarrow \operatorname{cf}^{V_{2}}(\beta) \geq \sigma .
$$

(Of course, also $\mathrm{cf}^{V_{1}}(\beta) \geq \sigma$.)

Now, looking at all $\alpha<\kappa^{+}$simultaneously, had we in our definition of $\left\langle E_{\alpha}: \alpha<\kappa^{+}\right\rangle$in the proof of (1) replaced each $E_{\alpha}$ by $E_{\alpha}^{\dagger}$, (i)-(iii) would still have been satisfied. So, by (**), had we used $E_{\alpha}^{\dagger}$ rather than $E_{\alpha}$ in the definition of $d_{\alpha}^{\dagger}$, we would have had

$$
\beta \in \operatorname{nacc}\left(d_{\alpha}^{\dagger}\right) \Rightarrow \operatorname{cf}^{V_{2}}(\beta) \geq \sigma
$$

as desired.

It only remains to check that $C^{*}$ would still satisfy (1), which is easy.

(3) Similar to (2). $\mathbf{-}_{2.0}$

Remark 2.1. (0) Note that a particular case of (iv) of Theorem 2.0 is that $V_{1} \vDash 2^{\kappa}=\kappa^{+}$.

(1) In fact, (iv) could be weakened using [Sh 430, §1].

Now we shall see that the same conclusion as in Theorem 2.0(1) can be obtained under somewhat different assumptions. We have no assumptions on $\operatorname{cf}\left(\mathrm{Club}^{V_{1}}(\kappa), \supseteq\right)$ or $\kappa^{+}$, but we have to add some assumptions on certain covering numbers. If we use forcing to go from $V_{1}$ to $V_{2}$, these correspond to the chain condition of the forcing used. We also do not need to require that $\kappa$ is a limit cardinal of $V_{1}$.

Theorem 2.2. Assume that:

(i) $V_{1} \subseteq V_{2}$ are models of $Z F C, V_{1}$ is a class of $V_{2}$ containing all ordinals and $V_{1}, V_{2}$ are transitive.

(ii) $V_{1} \vDash " \kappa$ is regular $>\aleph_{0}$ ".

(iii) $V_{2} \vDash " \operatorname{cf}(\kappa)=\theta, \kappa$ is a cardinal and $\theta<\kappa "$.

Moreover, assume that:

Either $\theta>\aleph_{0}$ and:

(iv $^{\prime}$ ) If $a \in V_{2}$ is such that

$$
V_{2} \vDash "|a| \leq \operatorname{cf}\left(\operatorname{Club}^{V_{2}}(\theta), \supseteq\right) " \quad \text { and } \quad V_{2} \vDash " a \subseteq \operatorname{Club}^{V_{1}}(\kappa) ",
$$


then we can find a sequence $\left\langle b_{i}: i<\theta\right\rangle \in V_{2}$ such that $\bigwedge_{i<\theta} b_{i} \in V_{1}$, while $V_{1} \vDash "\left|b_{i}\right| \leq \kappa$ " and $V_{2} \vDash " a \subseteq \bigcup_{i<\theta} b_{i}$ ". (Hence, $V_{2} \vDash " \operatorname{cf}\left(\operatorname{Club}^{V_{2}}(\theta), \supseteq\right) \leq$ $\kappa "$.

$\operatorname{Or} \theta=\aleph_{0}$ and:

$\left(\mathrm{iv}^{\prime \prime}\right) V_{2} \vDash " 2^{\aleph_{0}}<\kappa "$.

$\left(\mathrm{v}^{\prime \prime}\right)$ For every $a \subseteq \mathrm{Club}^{V_{1}}(\kappa)$ such that $|a|^{V_{2}} \leq 2^{\theta}$, there is a $b \in V_{1}$ such that $a \subseteq b$ and $|b|^{V_{1}} \leq \kappa$.

Then in $V_{2}$, we can find an unbounded $C^{*} \subseteq \kappa$ such that

$$
E \in \operatorname{Club}^{V_{1}}(\kappa) \Rightarrow C^{*} \backslash E \text { is bounded. }
$$

Before proving this theorem, let us make some remarks.

Remark 2.3. (0) Suppose that the first three assumptions of 2.2 are satisfied.

If $\operatorname{cf}\left(\mathrm{Club}^{V_{2}}(\theta), \supseteq\right)<\kappa$ and $V_{2}$ is obtained from $V_{1}$ by changing the cofinality of $\kappa$ to $\theta$ via a $\kappa^{+}$-cc forcing, then (iv') holds (in either of the two cases of the theorem). Actually, (iv') holds under some weaker conditions (see [Sh 410, §2] for this).

(1) It is also meaningful to use the notion of $\operatorname{cf}(\operatorname{Club}(\theta), \supseteq)$ for $\theta=$ $\aleph_{0}$. Namely, a club subset of $\omega$ is simply any unbounded set, and then $\operatorname{cf}\left(\operatorname{Club}\left(\aleph_{0}\right), \supseteq\right)$ corresponds to the familiar notion of $\mathbf{d}$, the smallest cardinality of a dominating family in $\left({ }^{\omega} \omega, \leq^{*}\right)$. If $\mathbf{d}=2^{\aleph_{0}}$, then obviously (iv $\left.{ }^{\prime}\right)$ is weaker than $\left(\mathrm{v}^{\prime \prime}\right)$.

(2) If $V_{1} \vDash " \operatorname{cf}(\operatorname{Club}(\kappa), \supseteq)=\kappa^{+}$" (as in Theorem 2.0), then $\left(\mathrm{v}^{\prime \prime}\right)$ holds. The natural case that we have in mind is $V_{2} \vDash$ " $2^{\theta}<\kappa$ ". So, if we are using a $\kappa^{+}$-cc forcing to pass from $V_{1}$ from $V_{2}$ and $V_{2} \vDash$ " $2^{\theta}<\kappa$ ", Theorem 2.2 is stronger than 2.0(1).

(3) Note that if $V_{1} \vDash " \kappa=\mu^{+} \& \mu$ is regular" then $V_{2} \vDash " \operatorname{cf}(\kappa)=\operatorname{cf}(\mu)$ ", by [Sh g, VII, §4] $=$ [Sh b, XIII, §4].

(4) As we mentioned earlier, a different proof of 2.2 can be found in [Gi1], with somewhat stronger assumptions in the case of $\theta>\aleph_{0}$ (that is, assuming $\left.2^{\theta}<\kappa\right)$.

We proceed to the proof of 2.2 .

Proof of 2.2. We break the proof into two cases:

Case 1: $\theta>\aleph_{0}$. Let $d=\left\{\alpha_{\zeta}: \zeta<\theta\right\} \in V_{2}$ be a club of $\kappa$. For each club $A \subseteq \theta$ from $V_{2}$, we try to set $C^{*}=d_{A}:=\left\{\alpha_{\zeta}: \zeta \in A\right\}$ and obtain $C^{*}$ which satisfies the theorem. If we succeed for some $A$, the theorem is proved. Note that each $d_{A}$ is unbounded in $\kappa$.

Otherwise, for each $A$ as above, we can choose an $E_{A} \in \mathrm{Club}^{V_{1}}(\kappa)$, which witnesses that $C^{*}=d_{A}$ does not work. So, in $V_{2}$ we have $\theta=\sup \{\zeta \in A$ : 
$\left.\alpha_{\zeta} \notin E_{A}\right\}$. Without loss of generality, each $E_{A}$ is a club subset of $\kappa$ in $V_{1}$.

Let $\mathcal{P} \subseteq \operatorname{Club}^{V_{2}}(\theta)$ be cofinal in $\left(\operatorname{Club}^{V_{2}}(\theta), \supseteq\right)$, of cardinality $\operatorname{cf}^{V_{2}}(\operatorname{Club}(\theta))$. Therefore $a:=\left\{E_{A}: A \in \mathcal{P}\right\}$ is a subset of $\mathrm{Club}^{V_{1}}(\kappa)$ of cardinality $\leq \operatorname{cf}^{V_{2}}(\operatorname{Club}(\theta), \supseteq)$. So we can find $\left\langle b_{i}: i<\theta\right\rangle$ as guaranteed by $\left(\mathrm{iv}^{\prime}\right)$.

In $V_{1}$, for $i<\theta$, let $\left\{C: C \in b_{i}\right.$ and $C$ is a club of $\left.\kappa\right\}$ be enumerated as $\left\{C_{j}^{i}: j<j_{i} \leq \kappa\right\}$ and let $E_{i}:=\Delta_{j<j_{i}} C_{j}^{i}$. In $V_{2}$, the set $E_{i} \cap d$ is a club of $\kappa$. Therefore, $A_{i}:=\left\{\zeta<\theta: \alpha_{\zeta} \in E_{i}\right\}$ is a club of $\theta$.

Now consider $A^{*}:=\Delta_{i<\theta} A_{i}=\left\{\zeta<\theta: \bigwedge_{i<\zeta} \alpha_{\zeta} \in E_{i}\right\}$. It is a club of $\theta$, so we can find an $A^{\otimes} \in \mathcal{P}$ such that $A^{\otimes} \subseteq A^{*}$. Without loss of generality, $A^{\otimes}$ is a club. So, $E_{A^{\otimes}}$ is well defined, and for some $i<\theta, E_{A^{\otimes}} \in b_{i}$, so $E_{A \otimes}=C_{j}^{i}$ for some $j<\kappa$. Fix such $i$ and $j$.

Then by the definition of $E_{i}$ and $A^{*}$, we have

$$
\zeta \in A^{*} \& \zeta>i \& \alpha_{\zeta}>j \Rightarrow \alpha_{\zeta} \in E_{A_{\otimes}},
$$

which is a contradiction with $A^{\otimes} \subseteq A^{*}$ and $\theta=\sup \left\{\zeta \in A^{\otimes}: \alpha_{\zeta} \notin E_{A \otimes}\right\}$.

Case 2: $\theta=\aleph_{0}$. As before, we first fix (in $V_{2}$ ) an increasing unbounded sequence $d=\left\{\alpha_{n}: n \in \omega\right\}$ in $\kappa$. For all $E \in \operatorname{Club}^{V_{1}}(\kappa)$, we try setting

$$
C^{*}=C^{*}(E)=\left\{\sup \left(\alpha_{n} \cap E\right): n \in \omega\right\} .
$$

Note that each $C^{*}(E)$ is unbounded in $\kappa$. If we succeed for some $E$, then we are done. So, from now on we assume otherwise.

To do the proof, we need the following fact.

FACT 2.2.a. If $\left\langle E_{i}: i<i^{*}<\left(2^{\aleph_{0}}\right)^{+}\right\rangle \in V_{2}$ is such that each $E_{i} \in$ $\operatorname{Club}^{V_{1}}(\kappa)$, then we can find an $E \in \operatorname{Club}^{V_{1}}(\kappa)$ such that

$$
\bigwedge_{i<i^{*}} \sup \left(E \backslash E_{i}\right)<\kappa
$$

Proof. Without loss of generality, each $E_{i}$ is a club of $\kappa$ in $V_{1}$. Now consider $a:=\left\{E_{i}: i<i^{*}\right\}$, and apply $\left(\mathrm{v}^{\prime \prime}\right)$ to find a $b \in V_{1}$ such that $|b|^{V_{1}} \leq \kappa$ and $a \subseteq b$. In $V_{1}$, by intersecting $b$ with the family of all clubs of $\kappa$ in $V_{1}$ if necessary, we can without loss of generality assume that each element of $b$ is a club of $\kappa$ in $V_{1}$. So, let us in $V_{1}$ enumerate $b$ as $b=\left\{C_{\varepsilon}: \varepsilon<\kappa\right\}$. Then let $E:=\Delta_{\varepsilon<\kappa} C_{\varepsilon}$, so $E \in \operatorname{Club}^{V_{1}}(\kappa)$. It is easily seen that $E$ is as required.

Continuation of the proof of 2.2 , Case 2. We place ourselves in $V_{2}$ and choose by induction on $i<\left(2^{\aleph_{0}}\right)^{+}$an $E_{i} \in \operatorname{Club}^{V_{1}}(\kappa)$ such that:

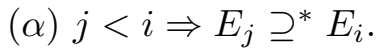

( $\beta) E_{i+1} \subseteq E_{i}$ shows that setting $C^{*}=C^{*}\left(E_{i}\right)$ does not give a satisfactory definition of $C^{*}$. 
This induction is easily carried out: at the successor stages we do as in $(\beta)$, and at the limit stages we use Fact 2.2.a.

For a given $i<\left(2^{\aleph_{0}}\right)^{+}$, if $n \in \omega$ is such that $\sup \left(\alpha_{n} \cap E_{i}\right) \notin E_{i+1}$, then $\sup \left(\alpha_{n} \cap E_{i}\right)>\sup \left(\alpha_{n} \cap E_{i+1}\right)$. Then it follows from $(\beta)$ that

$$
\bigwedge_{i<\left(2^{\aleph_{0}}\right)^{+}}\left\{n \in \omega: \sup \left(\alpha_{n} \cap E_{i+1}\right)<\sup \left(\alpha_{n} \cap E_{i}\right)\right\} \text { is infinite. }
$$

Now we define for each $i<\left(2^{\aleph_{0}}\right)^{+}$, a function $h_{i}: \omega \rightarrow \kappa$ by

$$
h_{i}(n)=\sup \left(\alpha_{n} \cap E_{i}\right) .
$$

So we have:

$(\alpha)^{*} j<i<\left(2^{\aleph_{0}}\right)^{+} \Rightarrow h_{j} \leq_{J_{\omega}^{\mathrm{bd}}} h_{i}$.

$(\beta)^{*} h_{i+1} \neq h_{i}\left(\bmod J_{\omega}^{\mathrm{bd}}\right)$.

This is a contradiction.

Similar in its proof to Theorem 2.2, the following theorem enables us to do outside guessing of clubs using a proper filter in $V_{2}$.

Theorem 2.4. Assume that:

(i) $V_{1} \subseteq V_{2}$ are transitive classes containing the ordinals and satisfying $Z F C$.

(ii) $V_{1} \vDash " \kappa$ is a regular cardinal $>\aleph_{0}$ ".

(iii) $V_{2} \vDash$ " $\kappa$ is a singular cardinal, $\operatorname{cf}(\kappa)=\theta$ ".

(iv) $V_{2} \vDash " \theta>\aleph_{0}$ ".

(v) $V_{1} \vDash " \mu=\operatorname{cf}(\operatorname{Club}(\kappa), \supseteq) "$.

(vi) $\sigma$ is a cardinal in $V_{2}$ and

$$
(\forall a)\left[a \in V_{2} \& a \subseteq \mu \&|a|<\sigma \rightarrow\left(\exists b \in V_{1}\right)(a \subseteq b \subseteq \mu \&|b|<\kappa)\right] .
$$

Then we can find in $V_{2}$ an increasing continuous sequence $\left\langle\alpha_{\zeta}^{*}: \zeta<\theta\right\rangle$ with limit $\kappa$, and a proper $\sigma$-complete filter $\mathcal{D}$ on $\theta$, such that

$(* * *) \quad$ For every club $E$ of $\kappa$ from $V_{1}$, we have $\left\{\zeta: \alpha_{\zeta+1}^{*} \in E\right\} \in \mathcal{D}$.

Remark 2.5. (0) If $\sigma=\aleph_{0}$, (vi) is redundant.

(1) The assumptions imply that $\sigma \leq \theta$. Otherwise we could use (vi) on a cofinal $\theta$-sequence of $\kappa$ in $V_{2}$, and obtain a contradiction with $V_{1} \vDash$ " $\kappa$ is regular".

(2) If instead of (vi) we have other properties of the style of [Sh 355, §5], or $\left(\right.$ iv $\left.^{\prime}\right)$ of Theorem 2.2, then we get corresponding completeness properties of the filter. For example, we could have that among any $\varrho$ members of $\mathcal{D}$, there are $\lambda$ whose intersection is also in $\mathcal{D}$, for some cardinal $\varrho \geq \lambda$.

(3) Remark 2.3(3) applies here as well. 
Proof of 2.4. Once we define $\left\langle\alpha_{\zeta}^{*}: \zeta<\theta\right\rangle$, we shall have

$\mathcal{D}=\left\{A \subseteq \theta\right.$ : for some $E \in \mathrm{Club}^{V_{1}}(\kappa)$, we have $\left.\left(\alpha_{\zeta+1}^{*} \in E \Rightarrow \zeta \in A\right)\right\}$.

This definition makes sense for any $\left\langle\alpha_{\zeta}^{*}: \zeta<\theta\right\rangle$ increasing continuous with limit $\kappa$ and yields a $\sigma$-complete filter $\mathcal{D}$ (by (vi)), and (***) holds. The point is to have $\mathcal{D}$ proper, i.e. $\emptyset \notin \mathcal{D}$, and we now show how to make the choice of $\left\langle\alpha_{\zeta}^{*}: \zeta<\theta\right\rangle$ which will satisfy this.

In $V_{2}$ we let $\left\{\xi_{\varepsilon}: \varepsilon<\theta\right\}$ be a strictly increasing cofinal sequence in $\kappa$. For each club $E$ of $\kappa$ from $V_{1}$, let

$$
d_{E}:=\left\{\sup \left(\xi_{\varepsilon} \cap E\right): \varepsilon<\theta \& E \cap\left(\xi_{\varepsilon}, \xi_{\varepsilon+1}\right) \neq \emptyset \& \xi_{\varepsilon}>\min (E)\right\} .
$$

So, every $d_{E}$ is an unbounded subset of $\kappa$. If for some $E$, an increasing enumeration of $d_{E}$ can be used for $\left\langle\alpha_{\zeta}^{*}: \zeta<\theta\right\rangle$, and a proper filter is obtained, we are done.

Otherwise, let $\left\{E_{\alpha}: \alpha<\mu\right\}$ be a cofinal sequence of clubs of $\kappa$ in $V_{1}$ and assume that the enumeration is in $V_{1}$. By induction on $n \in \omega$, we choose $\alpha_{n}<\mu$ such that:

- $\alpha_{0}=0$.

- $\alpha_{n+1}$ is such that $E_{\alpha_{n+1}}$ is a club exemplifying the failure of $d_{E_{\alpha_{n}}}$ to give a satisfactory definition of $\left\langle\alpha_{\zeta}^{*}: \zeta<\theta\right\rangle$. Without loss of generality, $E_{\alpha_{n+1}} \subseteq E_{\alpha_{n}}$.

Then $E^{*}=\bigcap_{n \in \omega} E_{\alpha_{n}}$ is an element of $V_{2}$, and a club of $\kappa$, so necessarily

$$
\theta=\sup \left\{\varepsilon:\left(\xi_{\varepsilon}, \xi_{\varepsilon+1}\right) \cap E^{*} \neq \emptyset\right\} .
$$

Note that the definition of $E_{\alpha_{n+1}}$ implies the existence of an $\varepsilon_{n}(*)<\theta$ such that

$$
\begin{aligned}
\varepsilon \in\left(\varepsilon_{n}(*), \theta\right) \&\left(\xi_{\varepsilon}, \xi_{\varepsilon+1}\right) \cap E_{\alpha_{n+1}} & \neq \emptyset \\
\Rightarrow \sup \left(\left(\xi_{\varepsilon}, \xi_{\varepsilon+1}\right)\right. & \left.\cap E_{\alpha_{n+1}}\right)<\sup \left(\left(\xi_{\varepsilon}, \xi_{\varepsilon+1}\right) \cap E_{\alpha_{n}}\right) .
\end{aligned}
$$

Now $\bigcup_{n \in \omega} \varepsilon_{n}(*)<\theta$, so take an $\varepsilon \in\left(\bigcup_{n \in \omega} \varepsilon_{n}(*), \theta\right)$ such that $\left(\xi_{\varepsilon}, \xi_{\varepsilon+1}\right) \cap$ $E^{*} \neq \emptyset$. Then the sequence $\left\langle\sup \left(\left(\xi_{\varepsilon}, \xi_{\varepsilon+1}\right) \cap E_{\alpha_{n}}\right): n \in \omega\right\rangle$ is a strictly decreasing sequence of ordinals, a contradiction.

3. The family $I_{<f}[\lambda]$. Throughout this paper, we dealt with sets with squares, some weak version of squares, and the ideal $I[\lambda]$. In this section we would like to explore the connection between these notions. We show that the notion of square, the one of the weak square, and the ideal $I[\lambda]$ are all definable by the same definition, simply by changing a certain parameter. We shall also discuss general properties of notions which can be defined in this way.

We now review some familiar notions and related families of sets. 
To avoid trivialities, in the following, $\lambda$ is always assumed to be a regular uncountable cardinal.

Definition 3.0. (0) For a subset $S^{+}$of $\lambda$, we say that $S^{+}$has a square if there is a sequence $\left\langle C_{\delta}: \delta \in S^{+}\right\rangle$such that:

(a) $C_{\delta}$ is a closed subset of $\delta$.

(b) $\beta \in C_{\delta} \Rightarrow \beta \in S^{+} \& C_{\beta}=\beta \cap C_{\delta}$.

(c) $\beta$ is a limit ordinal $\in S^{+} \Leftrightarrow \beta=\sup \left(C_{\beta}\right)$.

(d) $\operatorname{otp}\left(C_{\delta}\right)<\delta$.

$I^{\square}[\lambda]$ is the family of all subsets $S$ of $\lambda$ for which there is an $S^{+} \subseteq \lambda$ which has a square and $S \backslash S^{+}$is nonstationary.

(1) A subset $S$ of $\lambda$ is said to have a weak square if $S \subseteq S^{+}(\bmod \operatorname{Club}(\lambda))$ for some $S^{+} \subseteq \lambda$ and there is a sequence $\left\langle\mathcal{P}_{\delta}: \delta \in S^{+}\right\rangle$such that:

(a) Each $\mathcal{P}_{\delta}$ is a family of closed subsets of $\delta$, and if $\delta$ is a limit ordinal, all members of $\mathcal{P}_{\delta}$ are unbounded in $\delta$.

(b) $\beta \in a \in \mathcal{P}_{\delta} \Rightarrow a \cap \beta \in \mathcal{P}_{\beta}$.

(c) $\left|\mathcal{P}_{\delta}\right| \leq|\delta|$.

(d) $a \in \mathcal{P}_{\delta} \Rightarrow \operatorname{otp}(a)<\delta$.

$I^{\mathrm{w} \square}[\lambda]$ is the family of all subsets of $\lambda$ which have a weak square.

(2) For an $S \subseteq \lambda$, we say that $S$ is $\operatorname{good}$ if $S \subseteq S^{+}(\bmod \operatorname{Club}(\lambda))$ for some $S^{+} \subseteq \lambda$ such that there is a sequence $\bar{C}=\left\langle C_{\delta}: \delta<\lambda\right\rangle$ for which:

(a) $C_{\delta}$ is a closed subset of $\delta$.

(b) If $\alpha \in \operatorname{nacc}\left(C_{\delta}\right)$, then $C_{\alpha}=\alpha \cap C_{\delta}$.

(c) If $\delta \in S^{+}$, then $\delta=\sup \left(C_{\delta}\right)$ and $\operatorname{otp}\left(C_{\delta}\right)=\operatorname{cf}(\delta)<\delta$.

(d) $\operatorname{nacc}\left(C_{\delta}\right)$ is a set of successor ordinals.

$I[\lambda]$ is the family of good subsets of $\lambda$.

R e m ark 3.1. (0) The notions of square and weak square are well known and were introduced by Jensen. The first appearance of $I[\lambda]$ is in [Sh 108], or [Sh 88a]. It was also considered in [Sh 351], [Sh 420] and elsewhere. We have already used $I[\lambda]$ in the first section. The definition we use differs from the original definition in [Sh 88a] for example, but the equivalence is proved in [Sh 420, 1.2]. It is shown in [Sh 88a, 3(1)] that $I[\lambda]$ is a normal ideal on $\lambda$. Under certain circumstances, like when $\lambda$ is the successor of a strong limit singular, the ideal $I[\lambda]$ contains a maximal set [Sh 108], and we have made use of this fact in the first section. For various further properties of $I[\lambda]$ see the above references.

(1) Obviously, $I[\lambda] \cap I^{\square}[\lambda] \cap I^{\mathrm{w} \square}[\lambda] \supseteq \mathrm{NS}[\lambda]$, and each of $I[\lambda], I^{\mathrm{w} \square}[\lambda]$ and $I^{\square}[\lambda]$ is closed under taking subsets.

(2) The notation $I^{\square}[\lambda]$ and $I^{\mathrm{w}} \square[\lambda]$ should not suggest that these families are necessarily ideals. 
(3) Note that what we have defined now as a square on $S$ differs from the definition we used in $\S 1$. This does not have any effect on $I_{1}[\lambda]$ (see below), so we adopt the present definition for convenience.

We now introduce a notion which as its particular cases includes $I[\lambda]$, $I^{\square}[\lambda]$ and $I^{\mathrm{w} \square}[\lambda]$.

Definition 3.2. (0) Let $\lambda$ be a regular uncountable cardinal, and $f$ a function from $\lambda$ to the cardinals. To avoid trivialities, we assume that $f(i) \geq 2$ for all $i \in \lambda$.

We define $I_{<f}[\lambda]$ as the family of all $S \subseteq \lambda$ for which there is an $S^{+} \subseteq \lambda$ such that $S \backslash S^{+}$is nonstationary, and a sequence $\bar{C}=\left\langle C_{\delta}: \delta \in S^{+}\right\rangle$which has the following properties:

(a) $C_{\delta}$ is a closed subset of $\delta$.

(b) $\operatorname{otp}\left(C_{\delta}\right)<\delta$.

(c) $\delta \in S^{+}$is a limit ordinal $\Rightarrow \delta=\sup \left(C_{\delta}\right)$.

(d) $\operatorname{nacc}\left(C_{\delta}\right)$ is a set of successor ordinals.

(e) For every $\beta<\lambda$, the set

$$
\mathcal{P}_{\beta}=\mathcal{P}_{\beta}[\bar{C}]:=\left\{C_{\delta} \cap \beta: \beta \in C_{\delta}\right\}
$$

has cardinality $<f(\beta)$.

(1) We call $\bar{C}$ and $S^{+}$as above witnesses for $S \in I_{<f}[\lambda]$. Or, we say that they exemplify that $S \in I_{<f}[\lambda]$.

(2) If $f(i)=g(i)^{+}$for all $i$, we let

$$
I_{g}[\lambda]=I_{<f}[\lambda]
$$

If $f$ is constantly equal to $\mu$, we write $I_{<\mu}[\lambda]$ (or $I_{\mu}[\lambda]$ ) instead of $I_{<f}[\lambda]$ (or $\left.I_{f}[\lambda]\right)$.

Remark 3.3. (0) It is easy to see that for any choice of $f$, the family $I_{<f}[\lambda]$ includes all nonstationary subsets of $\lambda$, and that $I_{<f}[\lambda]$ is closed under taking subsets.

(1) Again, $I_{<f}[\lambda]$ is not always an ideal, and below we shall discuss sufficient conditions for $I_{<f}[\lambda]$ to be an ideal, or a normal ideal.

(2) By (b)-(c) of Definition 3.2(0), if $S \in I_{<f}[\lambda]$, then $S \cap$ REG is not stationary.

(3) Notice that if $f$ and $g$ are functions from $\lambda$ into the cardinals $\geq 2$, and $g(i) \leq f(i)$ for all $i$, then $I_{<g}[\lambda] \subseteq I_{<f}[\lambda]$.

We first make some general remarks about $I_{<f}[\lambda]$ which show to what extent we can generalize results about $I[\lambda]$ to this notion. The following notation will be convenient, and corresponds to the one introduced in [Sh $420]$ to study $I[\lambda]$. 
Notation 3.4. Suppose that $\lambda$ is a cardinal, $S \subseteq \lambda$ and $f$ is a function from $\lambda$ into the cardinals. The symbol $-\oplus_{\overline{\mathcal{P}}, S^{+}}^{f}(S)$ means that $S^{+} \subseteq \lambda$ and $\overline{\mathcal{P}}=\left\langle\mathcal{P}_{\alpha}: \alpha \in \lambda\right\rangle$ is a sequence of sets with the following properties:

(i) $\mathcal{P}_{\alpha}$ consists of $<f(\alpha)$ subsets of $\alpha$.

(ii) If $\delta \in S^{+}$is a limit ordinal, then there is an $x \subseteq \delta$ such that

$$
\operatorname{otp}(x)<\delta=\sup (x) \& \bigwedge_{\zeta<\delta} x \cap \zeta \in \bigcup_{\gamma<\delta} \mathcal{P}_{\gamma} .
$$

(iii) $S \backslash S^{+}$is nonstationary.

We formulate a lemma which corresponds to the fact [Sh 420, $\S 1]$ that the various definitions of $I[\lambda]$ considered in [Sh 88a], [Sh 108] and [Sh 420] are equivalent.

LEMma 3.5. Suppose that $\lambda$ is an uncountable regular cardinal and $f$ is a function from $\lambda$ into the cardinals. Let us enumerate the following statements:

(1) $S \in I_{<f}[\lambda]$.

$(2)-\oplus_{\overline{\mathcal{P}}, S^{+}}^{f}(S)$ for some $\overline{\mathcal{P}}$ and $S^{+}$such that all elements of $\bigcup_{\alpha<\lambda} \mathcal{P}_{\alpha}$ are closed.

(3) ${ }^{-} \oplus_{\overline{\mathcal{P}}, S^{+}}^{f}(S)$ for some $\overline{\mathcal{P}}$ and $S^{+}$.

(4) $-\oplus_{\overline{\mathcal{P}}, S^{+}}^{f}(S)$ for some $\overline{\mathcal{P}}$ and $S^{+}$such that all elements of $\bigcup_{\alpha<\lambda} \mathcal{P}_{\alpha}$ are closed and, for all limit $\delta \in S^{+}$, there is an $x \subseteq \delta$ with

$$
\operatorname{otp}(x)=\operatorname{cf}(\delta)<\delta=\sup (x) \& \bigwedge_{\zeta<\delta} x \cap \zeta \in \bigcup_{\alpha<\delta} \mathcal{P}_{\alpha} .
$$

(5) $S \in I_{<f}[\lambda]$, and this is witnessed by an $S^{+}$and $\left\langle C_{\delta}: \delta \in S^{+}\right\rangle$such that for all $\delta \in S^{+}$:

(i) $\operatorname{otp}\left(C_{\delta}\right)=\operatorname{cf}(\delta)$.

(ii) $\alpha \in \operatorname{nacc}\left(C_{\delta}\right) \Rightarrow \alpha \in S^{+} \& C_{\alpha}=C_{\delta} \cap \alpha$.

Then $(1) \Rightarrow(2) \Rightarrow(3)$ and $(4) \Rightarrow(2)$ and $(5) \Rightarrow(1)$ and $(5) \Rightarrow(4)$.

If we in addition assume that $f$ satisfies, for all $\beta \in \lambda$,

$$
\lambda \geq f(\beta) \geq \operatorname{cf}(f(\beta))>\max \left\{|\beta|, \aleph_{0}\right\},
$$

then $(3) \Rightarrow(5)$ (so in this case all notions (1)-(5) are equivalent).

Proof. $(1) \Rightarrow(2)$ and $(5) \Rightarrow(4)$. If $S^{+}$and $\left\langle C_{\delta}: \delta \in S^{+}\right\rangle$witness that $S \in I_{<f}[\lambda]$, then we can set $\overline{\mathcal{P}}=\overline{\mathcal{P}}[\bar{C}]$, i.e.

$$
\mathcal{P}_{\alpha}=\left\{C_{\beta} \cap \alpha: \alpha \in C_{\beta}\right\},
$$

and obtain ${ }^{-} \oplus_{\overline{\mathcal{P}}, S^{+}}^{f}(S)$. We also see that all elements of $\bigcup_{\alpha<\lambda} \mathcal{P}_{\alpha}$ are closed.

If $S^{+}$and $\bar{C}$ were chosen to witness (5), then $\overline{\mathcal{P}}$ witnesses (4). 
$(2) \Rightarrow(3)$ and $(5) \Rightarrow(1)$, as well as $(4) \Rightarrow(2)$, are obvious.

The difficult step is to prove $(3) \Rightarrow(5)$, assuming $(*)$. Luckily, the proof is exactly like the "only if" part of [Sh 420, 1.2].

Let us from now on always assume that $f$ is a function from $\lambda$ into the cardinals, and $f(\alpha) \geq 2$ unless stated otherwise.

We now show that $I[\lambda], I^{\square}[\lambda]$ and $I^{\mathrm{w}} \square[\lambda]$ all occur as particular cases of $I_{<f}[\lambda]$, with the appropriate choice of the parameter $f$. Another choice of $f$ will yield the "silly square" of [Sh 355$]$.

THEOREM 3.6. (1) If

$$
f(\alpha)= \begin{cases}\lambda & \text { if } \alpha \text { is a limit ordinal }, \\ 1 & \text { otherwise }\end{cases}
$$

then $I_{f}[\lambda]=I[\lambda]$.

(2) $I_{1}[\lambda]=I^{\square}[\lambda]$.

(3) If $f$ is given by $f(i)=|i|$, then $I_{f}[\lambda]=I^{\mathrm{w} \square}[\lambda]$.

(4) $\lambda \backslash S_{\lambda}^{\text {in }} \in I_{\lambda}[\lambda]$.

Proof. (1) Suppose that $S \in I[\lambda]$, as witnessed by $S^{+}$and $\bar{C}=\left\langle C_{\delta}\right.$ : $\delta \in \lambda\rangle$. Then the same $S^{+}$together with $\left\langle C_{\delta}: \delta \in S^{+}\right\rangle$witness that $S \in I_{f}[\lambda]$. So, $I[\lambda] \subseteq I_{f}[\lambda]$.

For the other direction, suppose that $S \in I_{f}[\lambda]$, as is witnessed by $S^{+}$ and $\left\langle C_{\delta}: \delta \in S^{+}\right\rangle$. We can assume that $S^{+}$consists of limit ordinals only. For $\beta \in \lambda \backslash S^{+}$, we define

$$
C_{\beta}= \begin{cases}C_{\delta} \cap \beta & \text { if there is a } \delta \in S^{+} \text {such that } \beta \in C_{\delta}, \\
\emptyset & \begin{array}{l}
\text { and } \beta \text { is a successor, } \\
\text { otherwise. }
\end{array}\end{cases}
$$

This definition is well posed, since $f(\beta)=1$ for $\beta$ a successor.

Now $S^{+}$and $\left\langle C_{\delta}: \delta \in \lambda\right\rangle$ witness that $S \in I[\lambda]$.

We did not necessarily obtain a sequence such that $\operatorname{otp}\left(C_{\delta}\right)=\operatorname{cf}(\delta)$ for a club of $\delta$, but merely $\operatorname{otp}\left(C_{\delta}\right)<\delta$. It is well known that this suffices (see [Sh 420] or [Sh 108], [Sh 88a]).

(2)-(3) Both easily follow from the corresponding definitions.

(4) This is from [Sh 355]. We simply choose for every $\alpha<\lambda$ which is a regular cardinal a club $C_{\alpha}$ of $\alpha$ with $\operatorname{otp}\left(C_{\alpha}\right)=\operatorname{cf}(\alpha)<\alpha$ such that $\operatorname{nacc}\left(C_{\alpha}\right)$ contains only successor ordinals. Of course, note that $\{\alpha<\lambda$ : $\left.\alpha=\beta^{+}\right\}$is not stationary in $\lambda . \mathbf{m}_{3.6}$

Just from the definition of $I_{<f}[\lambda]$, if $S \in I_{<f}[\lambda]$ is a costationary set, it seems possible that for every $S^{+}$which exemplifies this, $S^{+} \backslash S$ is stationary. The following theorem shows that this is impossible.

Theorem 3.7. Suppose that $S \in I_{<f}[\lambda]$. Then there is a set $S^{+}$witnessing this and such that $S^{+}=S$ modulo a nonstationary set. 
Proof. Let us start with a set $T$ such that $T$ and $\overline{\mathcal{D}}=\left\langle D_{\alpha}: \alpha \in T\right\rangle$ witness that $S \in I_{<f}[\lambda]$. Let $E$ be a club of $\lambda$ such that $S \cap E \subseteq T$, and we enumerate $E$ as an increasing continuous sequence $\left\langle\alpha_{\zeta}: \zeta<\lambda\right\rangle$.

Now we set $S_{1}:=\left\{\zeta: \alpha_{\zeta} \in E \cap S\right\}$, and for $\zeta \in S_{1}$ we define $C_{\zeta}^{1}:=$ $\left\{\xi<\zeta: \alpha_{\xi} \in D_{\alpha_{\zeta}}\right\}$.

Noting that the set

$$
D:=\left\{\zeta: \zeta=\alpha_{\zeta}\right\}
$$

is a club of $\lambda$, we can check that $S_{1}=S(\bmod \mathrm{NS}[\lambda])$. It is also easily seen that $\left\langle C_{\zeta}^{1}: \zeta \in S_{1}\right\rangle$ satisfies 3.2(0)(a)-(e), except that it is possible that some elements of $\operatorname{nacc}\left(C_{\zeta}^{1}\right)$ are limit ordinals. So, we shall define for $\zeta \in S_{1}$,

$$
\begin{aligned}
C_{\zeta} & :=\left\{\varepsilon: \varepsilon \in \operatorname{acc}\left(C_{\zeta}^{1}\right)\right\} \cup\left\{\varepsilon+1: \varepsilon \in \operatorname{nacc}\left(C_{\zeta}^{1}\right)\right\} & & \text { if } \zeta=\sup \left(C_{\zeta}^{1}\right), \\
C_{\zeta+1} & :=\left\{\varepsilon: \varepsilon \in \operatorname{acc}\left(C_{\zeta}^{1}\right)\right\} \cup\left\{\varepsilon+1: \varepsilon \in \operatorname{nacc}\left(C_{\zeta}^{1}\right)\right\} & & \text { if } \zeta \neq \sup \left(C_{\zeta}^{1}\right) .
\end{aligned}
$$

We set $S^{+}:=\left\{\zeta \in S^{1}: \zeta=\sup \left(C_{\zeta}^{1}\right)\right\} \cup\left\{\zeta+1: \zeta>\sup \left(C_{\zeta}^{1}\right)\right\}$. Then $S^{+}$ and $\bar{C}:=\left\langle C_{\zeta}: \zeta \in S^{+}\right\rangle$are as required. $\mathbf{-}_{3.7}$

We give a sufficient condition for $I_{<f}[\lambda]$ to be a normal ideal:

TheOREM 3.8. Suppose that $f$ satisfies for each limit $\beta \in \lambda$,

$$
\operatorname{cf}[(f(\beta))]>\beta .
$$

Then $I_{<f}[\lambda]$ is a normal ideal on $\lambda$.

Proof. It can be easily checked that $I_{<f}[\lambda]$ is an ideal, we shall show that the ideal is normal.

Suppose that $S \subseteq \lambda$ is given such that $S \notin I_{<f}[\lambda]$, and $g$ is a regressive function on $S$. We shall assume that for all $\alpha \in \operatorname{Ran}(g)$ the set $S_{\alpha}:=$ $g^{-1}(\{\alpha\})$ is an element of $I_{<f}[\lambda]$ and obtain a contradiction. Note that these assumptions imply that $S \cap S_{\lambda}^{\text {in }}$ is not stationary, so without loss of generality $S$ does not contain any regular cardinals.

If $f(\alpha)>\lambda$ on a stationary subset $T$ of $S$, then as in the proof of 3.6(4), we can show that $T \in I_{<f}[\lambda]$. So without loss of generality (using the fact that $I_{<f}[\lambda]$ is an ideal), $(*)$ of 3.5 holds on $S$. We shall show that (3) of 3.5 holds for $S$, which is a contradiction. For $\alpha \in \operatorname{Ran}(g)$, let $S_{\alpha}^{+}$and $\overline{\mathcal{P}}^{\alpha}=\left\langle\mathcal{P}_{\delta}^{\alpha}: \delta<\lambda\right\rangle$ be such that ${ }^{-} \oplus_{\overline{\mathcal{P}}^{\alpha}, S_{\alpha}^{+}}^{f}\left(S_{\alpha}\right)$ holds. For $\delta<\lambda$ we define

$$
\overline{\mathcal{P}}_{\delta}:=\bigcup_{\alpha<\delta}\left\{x \cup \alpha: x \in \mathcal{P}_{\delta}^{\alpha}\right\} \cup\{\alpha\}
$$

and let $S^{+}:=\left\{\delta\right.$ : for some $\alpha<\delta$ we have $\left.\delta \in S_{\alpha}^{+}\right\}$and $\overline{\mathcal{P}}:=\left\langle\mathcal{P}_{\delta}: \delta<\lambda\right\rangle$. Then $\overline{\mathcal{P}}$ and $S^{+}$exemplify $-\oplus_{\overline{\mathcal{P}}, S^{+}}^{f}(S) \cdot \mathbf{-}_{3.8}$

The family $I_{<f}[\lambda]$ does not uniquely determine $f$, as follows from 
FACT 3.9. Suppose that $f$ and $g$ are functions from $\lambda$ into the cardinals $\geq 2$, and related to each other by the following:

$$
g(i)= \begin{cases}2 & \text { if } i \text { is not a limit ordinal, and } f(i) \leq \lambda, \\ \min \left\{f(i), \lambda^{+}\right\} & \text {otherwise. }\end{cases}
$$

Then $I_{<f}[\lambda]=I_{<g}[\lambda]$.

Pr o o f. Since $g \leq f$, certainly $I_{<g}[\lambda] \subseteq I_{<f}[\lambda]$.

The other direction is simply the proof of [Sh 420,1.2] phrased in the language of this section.

From the previous discussion we see that for many functions $f$, the family $I_{f}[\lambda]$ is either one of the known families, or simply obtainable as a Boolean combination of these. Now we give a very easy example of a function $f$ for which this cannot be said, provided $\lambda$ is strongly inaccessible.

FACT 3.10. Suppose that $\lambda$ is a strongly inaccessible cardinal and $f$ is given on $\lambda$ by

$$
f(\alpha)=2^{|\alpha|^{+}} .
$$

Then $I_{<f}[\lambda]$ is a normal ideal on $\lambda$, and SING $\cap \lambda \in I_{<f}[\lambda]$.

Proof. That $I_{<f}[\lambda]$ is a normal ideal on $\lambda$ follows from 3.7. We can choose for singular $\delta<\lambda$ any closed subset $C_{\delta}$ of $\delta$ with nacc $\left(C_{\delta}\right)$ containing only successor ordinals, making sure that $C_{\delta}$ is unbounded in $\delta$ if $\delta$ is a limit ordinal. Then obviously for $\beta<\lambda$,

$$
\left|\left\{C_{\delta} \cap \beta: \beta \in C_{\delta}\right\}\right| \leq 2^{|\beta|}<f(\beta) \cdot \mathbf{-}_{3.10}
$$

Concluding remarks 3.11. A similar discussion could be made with a different requirement on $\left\langle C_{\delta}: \delta \in S^{+}\right\rangle$from 4.2 . We could for example require less closure and more coherence. In this way we can recover the argument of [Sh 186, §3], for example. By a definition of similar nature we can define an ideal equivalent to $I[\lambda, \kappa)$ of [DjSh 545].

4. Appendix: More on $I[\lambda]$. Shortly after we submitted the (rest of the) paper for publication, we were able to prove additional two theorems on $I[\lambda]$, which both seem to fit with the third section of the paper. These two theorems are the content of this appendix.

TheOREM 4.0. Suppose that $\lambda$ is a regular cardinal, $\kappa<\lambda$ is regular, and for each cardinal $\sigma \in(\kappa, \lambda)$ there is a $\mathcal{P}_{\sigma} \subseteq[\sigma]^{<\kappa}$ such that $\left|\mathcal{P}_{\sigma}\right|<\lambda$ and

$$
a \in[\sigma]^{\kappa} \Rightarrow\left(\exists b \in[a]^{\kappa}\right)\left([b]^{<\kappa} \subseteq \mathcal{P}_{\sigma}\right)
$$


Then there is an $S \in I[\lambda]$ such that

$$
\begin{aligned}
& A(S):=\{\delta \in \lambda: \operatorname{cf}(\delta)=\theta \text { for some regular } \theta \in(\kappa, \lambda) \text { such that } \\
& \left.2^{<\kappa}<\theta \& S_{\kappa}^{\theta} \in I[\theta] \& S_{\kappa}^{\delta} \backslash S \text { is stationary in } \delta\right\}
\end{aligned}
$$

is nonstationary in $\lambda$.

P r o o f. The theorem will follow from Lemma 4.1. The proof of the lemma is along the lines of various proofs presented in [Sh 108] or [Sh 88a].

LEMma 4.1. Suppose that $\kappa<\lambda$ are regular cardinals satisfying the assumptions of 4.0. Then for any regular $\theta \in(\kappa, \lambda)$ such that $2^{<\kappa}<\theta$, and for every $S_{\theta} \in I[\theta]$ which is a subset of $S_{\kappa}^{\theta}$, there is an $S_{\lambda}=S_{\lambda}\left(\theta, S_{\theta}\right) \in I[\lambda]$ such that

$$
\begin{aligned}
B\left(S_{\theta}, S_{\lambda}\right):=\left\{\delta \in S_{\theta}^{\lambda}:\right. & \text { for every increasing continuous }\left\langle\alpha_{\delta, i}: i<\theta\right\rangle \\
& \text { with limit } \delta, \text { the set } \\
& \left.\left\{i<\theta: i \in S_{\theta} \text { but } \alpha_{\delta, i} \notin S_{\lambda}\right\} \text { is stationary in } \theta\right\}
\end{aligned}
$$

is not stationary in $\lambda$.

Proof of 4.1. Since $S_{\theta} \in I[\theta]$, there is a sequence $\left\langle D_{i}: i<\theta\right\rangle$ and a set $S^{+}$which witness this, according to Definition 3.0(2). Note that the conclusion of the lemma does not change if a nonstationary set is removed from $S_{\theta}$, so we shall for convenience assume that $S_{\theta} \subseteq S^{+}$.

Let $\chi$ be large enough compared to $\lambda$, say $\chi=\beth_{9}(\lambda)^{+}$. We start with an increasing continuous sequence $\bar{N}=\left\langle N_{i}: i<\lambda\right\rangle$ of elementary submodels of $\left\langle H(\chi), \in, \prec^{*}\right\rangle$, with the following properties:

(a) $\left|N_{i}\right|<\lambda$.

(b) $\left\{\lambda, \theta, \kappa, S_{\theta},\left\langle D_{i}: i<\theta\right\rangle, S^{+}, 2^{<\kappa}\right\} \in N_{0}$.

(c) $\bar{N} \uparrow(i+1) \in N_{i+1}$ for all $i<\lambda$.

(d) $N_{i} \cap \lambda$ is an ordinal for all $i<\lambda$.

(e) $\theta \subseteq N_{0}$.

Note that $(*)$ as we have it is in fact equivalent to the same statement in which $\sigma$ is allowed to be any ordinal $<\lambda$. We shall assume this version of $(*)$ for notational convenience. Then we can without loss of generality require that

(f) $\mathcal{P}_{N_{i} \cap \lambda} \cup\left\{\mathcal{P}_{N_{i} \cap \lambda}\right\} \subseteq N_{i+1}$ for all $i<\lambda$.

Let $E:=\left\{\delta<\lambda: N_{\delta} \cap \lambda=\delta\right\}$, so $E$ consists of limit ordinals and is a club of $\lambda$ with $E \cap(\theta+1)=\emptyset$. Define

$$
\begin{array}{r}
S_{\lambda}:=\{\delta \in E: \operatorname{cf}(\delta)<\delta \text { and there is an } A \subseteq \delta=\sup (A) \text { such that } \\
\left.\qquad \bigwedge_{\alpha<\delta} A \cap \alpha \in N_{\delta} \& \operatorname{otp}(A)=\operatorname{cf}(\delta)\right\} .
\end{array}
$$


Observation. $S_{\lambda} \in I[\lambda]$.

[Why? A more general proof is in fact given in [Sh 108], but here is a proof using Lemma 4.5 below. We simply set $\overline{\mathcal{R}}:=\left\langle\mathcal{R}_{\alpha}: \alpha<\lambda\right\rangle$, where $\mathcal{R}_{\alpha}:=N_{\alpha} \cap \mathcal{P}(\alpha)$. The $\mathcal{R}_{\alpha}$ here stand in place of $\mathcal{P}_{\alpha}$ in Lemma 4.5.]

Proof of 4.1 continued. Assume now that: $\delta \in \operatorname{acc}(E) \cap S_{\theta}^{\lambda}$. We want to show that $\delta \notin B\left(S_{\theta}, S_{\lambda}\right)$. Let $\left\langle\alpha_{\delta, i}: i<\theta\right\rangle$ be an increasing continuous enumeration of $E \cap \delta$. We shall show that $\left\{i<\theta: i \in S_{\theta}\right.$ but $\left.\alpha_{\delta, i} \notin S_{\lambda}\right\}$ is not stationary in $\theta$.

ObServation. Since $S_{\theta} \in I[\theta]$, we can find a sequence $\left\langle C_{\alpha_{\delta, i}}: i<\theta\right\rangle$ such that:

- $C_{\alpha_{\delta, i}}$ is a subset of $\alpha_{\delta, i}$.

- $\operatorname{otp}\left(C_{\alpha_{\delta, i}}\right) \leq \kappa$.

- $\beta \in C_{\alpha_{\delta, i}} \Rightarrow \beta=\alpha_{\delta, j}$ for some $j$ and $C_{\beta}=C_{\alpha_{\delta, i}} \cap \alpha_{\delta, j}$.

- $i \in S_{\theta} \Rightarrow \alpha_{\delta, i}=\sup \left(C_{\alpha_{\delta, i}}\right)$.

[How do we find such a sequence? First we set $C_{i}^{*}$ to be the first $\kappa$ nonaccumulation points of $D_{i}$, for $i<\theta$. Then let $C_{\alpha_{\delta, i}}:=\left\{\alpha_{\delta, j}: j \in C_{i}^{*}\right\}$.]

Proof of 4.1 continued. Note that the sequence $\left\langle C_{i}^{*}: i\langle\theta\rangle\right.$ is both an element and a subset of $N_{0}$. We also see that every $C_{\alpha_{\delta, i}}$ is in $N_{\delta}$, but note that we do not know that necessarily $C_{\alpha_{\delta, i}} \in N_{\alpha_{\delta, i}+1}$. So we shall define a function $h: \theta \rightarrow \theta$ by

$h(i):=\min \{\varepsilon<\theta:$

$$
\left.\left(\forall x \in\left[C_{i}^{*}\right]^{<\kappa}\right)\left(\left\{\alpha_{\delta, j}: j \in x\right\} \in N_{\delta} \Rightarrow\left\{\alpha_{\delta, j}: j \in x\right\} \in N_{\alpha_{\delta, \varepsilon}}\right)\right\} .
$$

Note that if $x \in\left[C_{i}^{*}\right]^{<\kappa}$ and $\left\{\alpha_{\delta, j}: j \in x\right\} \in N_{\delta}$, then $\left\{\alpha_{\delta, j}: j \in x\right\} \in N_{\alpha_{\delta, \varepsilon}}$ for some $\varepsilon<\theta$, as $2^{<\kappa}<\theta$. (So $h(i)$ is well defined for all $i<\theta$.)

[Why? We can find a $\xi<\delta$ such that $N_{\xi}$ already contains all bounded subsets of $\kappa$ that are going to appear in $N_{\delta}$, as otherwise we would be able to inductively construct $\theta$ many bounded subsets of $\kappa$, in contradiction with $2^{<\kappa}<\theta$.]

Let

$$
e:=\{i<\theta:(\forall j<i)(h(j)<i)\} .
$$

Obviously, $e$ is a club of $\theta$. We claim $S_{\theta} \cap e \subseteq\left\{i<\theta: \alpha_{\delta, i} \in S_{\lambda}\right\}$. (Hence, $\left\{i<\theta: i \in S_{\theta}\right.$ but $\left.\alpha_{\delta, i} \notin S_{\lambda}\right\}$ is not stationary in $\lambda$.)

To see this, consider an $\alpha_{\delta, i}$ for some $i \in S_{\theta} \cap e$. We know that $\alpha_{\delta, i} \in E$ and $\operatorname{cf}\left(\alpha_{\delta, i}\right)=\operatorname{cf}(i)=\kappa<\alpha_{\delta, i}$, as $i \in S_{\theta}$. Now, $C_{\alpha_{\delta, i}}$ is unbounded in $\alpha_{\delta, i}$, so $\operatorname{otp}\left(C_{\alpha_{\delta, i}}\right)=\kappa$. Therefore for some $A_{i} \subseteq C_{\alpha_{\delta, i}}$ we have

$$
\operatorname{otp}\left(A_{i}\right)=\kappa \&\left[A_{i}\right]^{<\kappa} \subseteq \mathcal{P}_{\alpha_{\delta, i}} .
$$

So $\sup \left(A_{i}\right)=\alpha_{\delta, i}$, and it suffices to see that for all $\alpha<\alpha_{\delta, i}$ we have $A_{i} \cap \alpha \in N_{\alpha_{\delta, i}}$. 
Let us fix an $\alpha<\alpha_{\delta, i}$. Let $j^{*}=\min \left\{j<i: \alpha \leq \alpha_{\delta, j} \in A_{i}\right\}$. Therefore

$$
A_{i} \cap \alpha=A_{i} \cap \alpha_{\delta, j^{*}}=A_{i} \cap C_{\alpha_{\delta, i}} \cap \alpha_{\delta, j^{*}}=A_{i} \cap C_{\alpha_{\delta, j^{*}}} .
$$

Let $x \subseteq C_{j^{*}}^{*}$ be such that $A_{i} \cap C_{\alpha_{\delta, j^{*}}}=\left\{\alpha_{\delta, j}: j \in x\right\}$, so $|x|<\kappa$. Note that by the choice of $A_{i}$ we have $A_{i} \cap \alpha \in \mathcal{P}_{\alpha_{\delta, i}}$. Since $\mathcal{P}_{\alpha_{\delta, i}} \subseteq N_{\alpha_{\delta, i}+1} \subseteq N_{\delta}$, we have $A_{i} \cap \alpha=\left\{\alpha_{\delta, j}: j \in x\right\} \in N_{\delta}$, therefore $A_{i} \cap \alpha \in N_{\alpha_{\delta}, h(j)}$. As $h(j)<i$, our claim is correct.

Consequently, $\delta \notin B\left(S_{\theta}, S_{\lambda}\right)$, so $B\left(S_{\theta}, S_{\lambda}\right) \cap \operatorname{acc}(E)=\emptyset$, hence $B\left(S_{\theta}, S_{\lambda}\right)$ is not stationary.

Proof of 4.0 continued. Suppose that $\kappa$ and $\lambda$ are as in the assumptions of the theorem, and $\theta \in(\kappa, \lambda)$ is regular and such that $2^{<\kappa}<\theta$ and $S_{\kappa}^{\theta} \in I[\theta]$. We will apply Lemma 4.1 to $\kappa, \theta$ and $\lambda$. By the lemma, we can find a set $S_{\lambda}(\theta) \in I[\lambda]$ such that $B\left(S_{\kappa}^{\theta}, S_{\lambda}(\theta)\right)$ is not stationary in $\lambda$.

Let $S$ be the diagonal union of $\left\{S_{\lambda}(\theta): S_{\lambda}(\theta)\right.$ is defined $\}$, i.e.

$$
S:=\left\{\alpha<\lambda:(\exists \theta<\alpha)\left(S_{\lambda}(\theta) \text { is defined and } \alpha \in S_{\lambda}(\theta)\right)\right\} .
$$

It follows from the normality of $I[\lambda]$ that it is closed under formation of such unions, so $S \in I[\lambda]$.

Now suppose that $A(S)$ is stationary in $\lambda$; then there must be a regular $\theta<\lambda$ such that $2^{<\kappa}<\theta$ and $S_{\kappa}^{\theta} \in I[\theta]$ and $\left\{\delta \in S_{\theta}^{\lambda}: S_{\kappa}^{\delta} \backslash S\right.$ is stationary in $\delta$ \} is stationary in $\lambda$. Note that $S_{\lambda}(\theta)$ is defined. Now, for any $\delta>\theta$ in the above set, $S_{\kappa}^{\delta} \backslash S_{\lambda}(\theta)$ is stationary in $\delta$. In particular, for every sequence $\left\langle\alpha_{\delta, i}: i<\theta\right\rangle$ which increasingly enumerates a club of $\delta$, the set $\{i<\theta$ : $\left.\operatorname{cf}(i)=\kappa \& \alpha_{\delta, i} \notin S_{\lambda}(\theta)\right\}$ is stationary in $\theta$. Hence $\delta \in B\left(S_{\kappa}^{\theta}, S_{\lambda}(\theta)\right)$, and the set of such $\delta$ is nonstationary in $\lambda$, by the lemma. This is a contradiction, hence $S$ is as required.

Remark and Conclusion 4.2. Property (*) of 4.0 was considered in [Sh 430]. Obviously, $(*)$ is implied by

$$
(\forall \sigma<\lambda)\left(\sigma^{<\kappa}<\lambda\right) \text {. }
$$

Also, $S_{\kappa}^{\theta} \in I[\theta]$ obviously follows from $(\forall \alpha<\theta)\left(|\alpha|^{\kappa}<\theta\right)$. Hence, if for example $\lambda \geq \beth_{\omega}$ and $\kappa<\beth_{\omega}$ are regular, there is a set $S \in I[\lambda]$ and an $n \in \omega$ such that for all regular $\theta \in\left(\beth_{n}, \lambda\right)$, the set $\left\{\delta \in S_{\theta}^{\lambda}: S_{\kappa}^{\delta} \backslash S\right.$ is stationary in $\delta$ \} is nonstationary in $\lambda$.

Definition 4.3. Let $\lambda$ be a regular uncountable cardinal.

(1) For $\Theta \subseteq$ REG, let

$$
S_{\Theta}:=\{\delta: \delta>\operatorname{cf}(\delta) \in \Theta\} .
$$

(2) A sequence $\left\langle\mathcal{C}_{\alpha}: \alpha<\lambda\right\rangle$ is said to be a $\Theta$-weak square on $S \subseteq \lambda$ iff there is an $S^{+} \subseteq \lambda$ such that $S \backslash S^{+}$is nonstationary, and for every $\alpha<\lambda$ :

(i) $\mathcal{C}_{\alpha}$ is a nonempty family of subsets of $\alpha$. 
(ii) $\left|\mathcal{C}_{\alpha}\right|<\lambda$.

(iii) $C \in \mathcal{C}_{\alpha} \Rightarrow C$ is closed.

(iv) $\emptyset \neq C \in \mathcal{C}_{\alpha} \& \alpha$ is a limit ordinal $\Rightarrow \alpha \in S^{+} \& \sup (C)=\alpha \& \operatorname{otp}(C)$ $<\min (C)$.

(v) $\beta \in C \& C \in \mathcal{C}_{\alpha} \& \beta \in S_{\Theta} \Rightarrow \beta \in S^{+} \& C \cap \beta \in \mathcal{C}_{\beta}$.

Theorem 4.4. Let $\lambda$ be a regular uncountable cardinal. Suppose that $S \in I[\lambda]$ and

$\Theta:=\{\theta<\lambda:$

$\operatorname{cf}(\theta)=\theta$ \& every tree with $<\lambda$ nodes has $<\lambda$ branches of length $\theta\}$.

Then there is a $\Theta$-weak square on $S$.

The following lemma was proved in [Sh 420]:

Lemma 4.5. Let us define $I^{\prime}[\lambda]$ as the family of all subsets $S$ of $\lambda$ for which there is an $S^{+}$and $\left\langle\mathcal{P}_{\alpha}: \alpha<\lambda\right\rangle$ such that $S \backslash S^{+}$is nonstationary and for every $\alpha \in \lambda$ :

(A) $\mathcal{P}_{\alpha}$ is a family of $<\lambda$ subsets of $\alpha$.

(B) If $\alpha \in S^{+}$, then there is an unbounded subset $a \subseteq \alpha$ such that $(\forall \beta \in \alpha)\left(a \cap \beta \in \mathcal{P}_{\beta}\right)$.

Then:

(1) $I^{\prime}[\lambda]=I[\lambda]$.

(2) Without loss of generality we can require in the definition of $I^{\prime}[\lambda]$ that

(C) The sets in $\bigcup_{\alpha<\lambda} \mathcal{P}_{\alpha}$ are closed and for $\alpha \in S^{+}$, the set a from (B) satisfies

$$
\operatorname{otp}(a)=\operatorname{cf}(a)<\min (a) .
$$

For completeness we shall include the proof of the first part of this lemma. We do not prove (2), as the proof is the same as that of 3.5.

Pro of of 4.5(1). It follows by 3.5 and 3.6(1) that $I^{\prime}[\lambda] \subseteq I[\lambda]$, so let us start with an $S \in I[\lambda]$ and show that $S \in I^{\prime}[\lambda]$. Let $\overline{\mathcal{P}}_{\alpha}=\left\langle\mathcal{P}_{\alpha}^{0}: \alpha<\lambda\right\rangle$ and $S^{+}$exemplify that $S \in I[\lambda]$. (We use the definition from [Sh 108].)

For $\alpha<\lambda$ let us define $\mathcal{P}_{\alpha}^{1}$ by

$$
\mathcal{P}_{\alpha}^{1}:=\left\{c \cap[\gamma, \beta): \gamma \leq \beta \leq \alpha \& c \in \mathcal{P}_{\alpha}^{0}\right\},
$$

and let

$$
\mathcal{P}_{\alpha}^{2}:=\bigcup_{\beta \leq \alpha} \mathcal{P}_{\beta}^{1}
$$

For every $a \in \bigcup_{\alpha<\lambda} \mathcal{P}_{\alpha}^{2}$, we define a function $f_{a}$ with $\operatorname{Dom}\left(f_{a}\right)=a$ by

$$
f_{a}(\gamma):=\min \left\{\beta \geq \gamma: a \cap \gamma \in \mathcal{P}_{\beta}^{2}\right\}
$$


Note that $f_{a}(\gamma)$ is well defined for any $\gamma \in a$. Finally, let

$$
\mathcal{P}_{\alpha}:=\left\{\operatorname{Ran}\left(f_{a}\right) \cap[\gamma, \beta): a \in \mathcal{P}_{\alpha}^{2} \& \gamma \leq \beta \leq \alpha\right\} .
$$

Let us check that $\overline{\mathcal{P}}:=\left\langle\mathcal{P}_{\alpha}: \alpha<\lambda\right\rangle$ and $S^{+}$exemplify that $S \in I^{\prime}[\lambda]$.

First we need to observe the following facts:

(1) $\gamma \leq f_{a}(\gamma)$ if $\gamma \in a \in \bigcup_{\alpha<\lambda} \mathcal{P}_{\lambda}^{2}$.

This follows just from the definition of $f_{a}$.

(2) $\gamma_{1} \leq \gamma_{2} \in a \in \bigcup_{\alpha<\lambda} \mathcal{P}_{\lambda}^{2} \Rightarrow f_{a}\left(\gamma_{1}\right) \leq f_{a}\left(\gamma_{2}\right)$.

Let $\beta_{1}:=f_{a}\left(\gamma_{1}\right)$. Suppose that $f_{a}\left(\gamma_{2}\right)<\beta_{1}$, so in particular there is a $\beta_{2}<\beta_{1}$ such that $a \cap \gamma_{2} \in \mathcal{P}_{\beta_{2}}^{2}$. By the definition of $\mathcal{P}_{\beta_{2}}^{2}$, there is some $\beta_{3} \leq \beta_{2}$ such that $a \cap \gamma_{2} \in \mathcal{P}_{\beta_{3}}^{1}$. Therefore $a \cap \gamma_{2}=b \cap\left[\beta_{4}, \beta_{5}\right)$ for some $\beta_{4} \leq \beta_{5} \leq \beta_{3}$ and $b \in \mathcal{P}_{\beta_{3}}^{0}$. In particular, $\gamma_{1} \in b \cap\left[\beta_{4}, \beta_{5}\right)$. Hence $b \cap\left[\beta_{4}, \gamma_{1}\right)=$ $a \cap \gamma_{1}$ is in $\mathcal{P}_{\beta_{3}}^{1}$, so in $\mathcal{P}_{\beta_{2}}^{2}$, which contradicts the minimality of $\beta_{1}$ in the definition of $f_{a}\left(\gamma_{1}\right)$.

(3) $\gamma \in a \Rightarrow f_{a \cap \gamma}=f_{a} \uparrow(a \cap \gamma)$.

First note that $f_{a \cap \gamma}$ is well defined an then just use the definition of $f_{a}$.

Now we check that $\overline{\mathcal{P}}$ has the required properties. It is obvious from the definition of $\overline{\mathcal{P}}$ that $\alpha<\lambda \Rightarrow \mathcal{P}_{\alpha} \subseteq \mathcal{P}(\alpha)$ and $\left|\mathcal{P}_{\alpha}\right|<\lambda$. Now suppose that $\alpha \in S^{+}$, and let $a$ be an unbounded subset of $\alpha$ with $\operatorname{otp}(a)<\alpha$ and

$$
(\forall \beta<\alpha)\left(a \cap \beta \in \bigcup_{\gamma<\alpha} \mathcal{P}_{\gamma}^{0}\right) .
$$

Notice that for every $\gamma<\lambda$ we have $\mathcal{P}_{\gamma}^{0} \subseteq \mathcal{P}_{\gamma}^{1} \subseteq \mathcal{P}_{\gamma}^{2}$, so $f_{a}$ is defined. By the choice of $a$ and $\alpha$ we see that $\operatorname{Ran}\left(f_{a}\right) \subseteq \alpha$. Moreover, by (1) above, $\operatorname{Ran}\left(f_{a}\right)$ is unbounded in $\alpha$. Since $\operatorname{Dom}\left(f_{a}\right)=a$, it follows that $\operatorname{otp}\left(\operatorname{Ran}\left(f_{a}\right)\right) \leq$ $\operatorname{otp}(\operatorname{Dom})\left(f_{a}\right)<\alpha$.

It remains to see that if $\beta \in \operatorname{Ran}\left(f_{a}\right)$, then $\beta \cap \operatorname{Ran}\left(f_{a}\right) \in \mathcal{P}_{\beta}$. By (1)(3) above we know that $\operatorname{Ran}\left(f_{a}\right) \cap \beta=\operatorname{Ran}\left(f_{a \cap \beta}\right)$, so we have proved our lemma. - $4.5(1)$

Proof of Theorem 4.4. Let $S^{+}$and $\left\langle\mathcal{P}_{\alpha}: \alpha<\lambda\right\rangle$ exemplify that $S \in I[\lambda]$. By the above lemma we can assume that for every $\alpha \in S^{+}$, there is an unbounded subset $a$ of $\alpha$ such that

$$
(\forall \beta<\alpha)\left(a \cap \beta \in \mathcal{P}_{\beta}\right) \& \operatorname{otp}(a)=\operatorname{cf}(a)<\min (a)
$$

and that each $\mathcal{P}_{\alpha}$ consists of closed sets. Let us define

$$
\mathcal{C}_{\alpha}= \begin{cases}\left\{a \subseteq \alpha:(\forall \beta<\alpha)\left(a \cap \beta \in \mathcal{P}_{\beta}\right)\right. \\ \& a \neq \emptyset \Rightarrow \operatorname{cf}(\alpha)=\operatorname{otp}(a)<\min (a) \\ \& \alpha \operatorname{limit} \Rightarrow \sup (a)=\alpha\} \quad \text { if } \alpha \in S_{\Theta} \cap S^{+} \text {or } \alpha \text { a successor } \\ \{\emptyset\} & \text { otherwise }\end{cases}
$$


Then it is easy to check all the requirements for a $\Theta$-weak square. That $\mathcal{C}_{\alpha}$ is never empty follows from the definition of $I[\lambda]$. To see that for $\alpha \in S_{\Theta}$ we have $\left|\mathcal{C}_{\alpha}\right|<\lambda$, consider the tree $T$ which is defined in the following way.

For $\beta<\alpha$, the $\beta$-level of $T$ is

$$
\operatorname{lev}_{\beta}(T):=\left\{a \cap \beta: a \in \mathcal{C}_{\alpha}\right\}
$$

and $T$ is ordered by $\subseteq$. Then $T$ has $<\lambda$ nodes, and every element of $\mathcal{C}_{\alpha}$ is the union of an $\alpha$-branch of $T$. As $\theta:=\operatorname{cf}(\alpha) \in \Theta$, by the definition of $\Theta$ we have $\left|\mathcal{C}_{\alpha}\right|<\lambda$.

Also note that all elements of $\mathcal{C}_{\alpha}$ are increasing unions of closed sets without the last element, so they are closed.

Finally, if $\beta \in C \& C \in \mathcal{C}_{\alpha} \& \beta \in S_{\Theta}$, then $\beta \in S^{+}$and $C \cap \beta \in \mathcal{C}_{\beta}$. $\mathbf{-}_{4.4}$

Remark 4.6. If $\lambda>\mu$ is regular and $\mu$ is a strong limit singular with $\kappa=\operatorname{cf}(\mu)$, then $\Theta=\operatorname{REG} \cap \mu \backslash\{\kappa\}$ satisfies the condition of Theorem 4.4, by [Sh $460,1.1]$.

\section{References}

[DjSh 545] M. Džamonja and S. Shelah, Saturated filters at successors of singulars, weak reflection and yet another weak club principle, submitted.

[Gi] M. Gitik, Some results on nonstationary ideal, Israel J. Math., to appear.

[Gi1] -, Some results on nonstationary ideal II, submitted.

[Sh b] S. Shelah, Proper Forcing, Lecture Notes in Math. 940, Springer, 1982.

[Sh e] -, Non Structure Theory, Oxford Univ. Press, to appear.

[Sh f] —, Proper and Improper Forcing, Oxford Univ. Press, to appear.

[Sh g] - Cardinal Arithmetic, Oxford Univ. Press, 1994.

[Sh 88a] -, Appendix: on stationary sets (to Classification of nonelementary classes II. Abstract elementary classes), in: Classification Theory (Chicago, IL, 1985), Proc. USA-Israel Conf. on Classification Theory, Chicago, December 1985, J. T. Baldwin (ed.), Lecture Notes in Math. 1292, Springer, 1987, $483-495$.

[Sh 108] -, On successors of singular cardinals, in: Logic Colloquium 78, M. Boffa, D. van Dalen and K. McAloon (eds.), North-Holland, 1979, 357-380.

[Sh 186] —, Diamonds, uniformization, J. Symbolic Logic 49 (1984), 1022-1033.

[Sh 237e] - Remarks on squares, in: Around Classification Theory of Models, Lecture Notes in Math. 1182, Springer, 1986, 276-279.

[Sh 351] -, Reflecting stationary sets and successors of singular cardinals, Arch. Math. Logic 31 (1991), 1-29.

[Sh 355] -, $\aleph_{\omega+1}$ has a Jonsson algebra, in: Cardinal Arithmetic, Chapter II, Oxford Univ. Press, 1994.

[Sh 365] -, Jonsson algebras in inaccessible cardinals, in: Cardinal Arithmetic, Chapter III, Oxford Univ. Press, 1994.

[Sh 400] -, Cardinal arithmetic, in: Cardinal Arithmetic, Chapter IX, Oxford Univ. Press, 1994.

[Sh 410] -, More on cardinal arithmetic, Arch. Math. Logic 32 (1993), 399-428. 
[Sh 420] S. Shelah, Advances in cardinal arithmetic, in: Finite and Infinite Combinatorics in Sets and Logic, W. W. Sauer et al. (eds.), Kluwer Acad. Publ., 1993, 355-383.

[Sh 430] —, Further cardinal arithmetic, Israel J. Math., to appear.

[Sh 460] -, The generalized continuum hypothesis revisited, ibid., submitted.

[Sh 580] —, Strong covering revisited, Fund. Math., to appear.

Current address of Mirna Džamonja:

INSTITUTE OF MATHEMATICS

HEBREW UNIVERSITY OF JERUSALEM

91904 GIVAT RAM, ISRAEL

E-mail: DZAMONJA@MATH.HUJI.AC.IL SHELAH@MATH.HUJI.AC.IL
MATHEMATICS DEPARTMENT UNIVERSITY OF WISCONSIN-MADISON MADISON, WISCONSIN 53706 U.S.A.

Received 23 February 1995;

in revised form 14 May 1995 